\title{
Gas Mass Tracers in Protoplanetary Disks: CO is Still the Best
}

\author{
Tamara Molyarova ${ }^{1,2}$ (1) , Vitaly Akimkin ${ }^{1}$ (1) , Dmitry Semenov $^{3}$, Thomas Henning ${ }^{3}$, Anton Vasyunin ${ }^{4,5}$ (1), and Dmitri Wiebe ${ }^{1}$ (1) \\ ${ }^{1}$ Institute of Astronomy, Russian Academy of Sciences, 48 Pyatnitskaya St., Moscow, 119017, Russia; molyarova@inasan.ru \\ ${ }^{2}$ Moscow Institute of Physics and Technology (State University), 9 Institutskiy per., Dolgoprudny, Moscow Region, 141700, Russia \\ ${ }^{3}$ Max Planck Institute for Astronomy, Königstuhl 17, D-69117 Heidelberg, Germany \\ ${ }^{4}$ Max Planck Institute for Extraterrestrial Physics, Giessenbachstrasse, D-85748 Garching, Germany \\ ${ }^{5}$ Ural Federal University, Ekaterinburg, 620083, Russia \\ Received 2017 June 14; revised 2017 October 6; accepted 2017 October 7; published 2017 November 8
}

\begin{abstract}
Protoplanetary disk mass is a key parameter controlling the process of planetary system formation. CO molecular emission is often used as a tracer of gas mass in the disk. In this study, we consider the ability of CO to trace the gas mass over a wide range of disk structural parameters, and we search for chemical species that could possibly be used as alternative mass tracers to CO. Specifically, we apply detailed astrochemical modeling to a large set of models of protoplanetary disks around low-mass stars to select molecules with abundances correlated with the disk mass and being relatively insensitive to other disk properties. We do not consider sophisticated dust evolution models, restricting ourselves to the standard astrochemical assumption of $0.1 \mu \mathrm{m}$ dust. We find that $\mathrm{CO}$ is indeed the best molecular tracer for total gas mass, despite the fact that it is not the main carbon carrier, provided reasonable assumptions about $\mathrm{CO}$ abundance in the disk are used. Typically, chemical reprocessing lowers the abundance of $\mathrm{CO}$ by a factor of 3, compared to the case where photodissociation and freeze-out are the only ways of $\mathrm{CO}$ depletion. On average, only 13\% $\mathrm{C}$ atoms reside in gas-phase $\mathrm{CO}$, albeit with variations from $2 \%$ to $30 \%$. $\mathrm{CO}_{2}, \mathrm{H}_{2} \mathrm{O}$, and $\mathrm{H}_{2} \mathrm{CO}$ can potentially serve as alternative mass tracers, with the latter two only applicable if disk structural parameters are known.
\end{abstract}

Key words: astrochemistry - circumstellar matter - protoplanetary disks

\section{Introduction}

A young Sun-like star at its early evolutionary stage is generally surrounded by a flattened rotating gas and dust structure, which is commonly referred to as a protoplanetary disk even though evidence of planet formation in these disks is still mostly circumstantial (see, e.g., reviews by Williams \& Cieza 2011; Armitage 2015). Although the exact scenario of planet formation remains debated, the mass of the protoplanetary disk is thought to play a key role in this process (Mordasini et al. 2012; Bitsch et al. 2015). Therefore, estimating accurate disk masses from observations is one of the most important but also most challenging tasks in studies of formation and early evolution of planetary systems.

There are two different approaches to measuring disk masses. The most common way is to observe the dust continuum emission. Provided this emission is optically thin (which is expected at submillimeter and millimeter wavelengths), one can calculate the total dust mass in the disk (Andrews \& Williams 2005; Williams \& Cieza 2011) from the integrated continuum flux. Then, to convert the dust mass into total disk mass, the standard interstellar gas-to-dust ratio of 100 can be adopted (Bohlin et al. 1978). However, to apply this method, one needs to know the dust temperature in the disk, because the same flux at a given wavelength may come from both a larger amount of cold dust or a smaller amount of warm dust. Such a mass derivation requires assumptions about dust optical properties, which are not well-constrained for dust grains in protoplanetary disks and also may change as dust grows at early stages of planet formation (Draine 2006; Henning \& Meeus 2011). Furthermore, it is far from obvious that the interstellar gas-to-dust mass ratio is valid for protoplanetary disks. Dunham et al. (2014) and Tsukamoto et al. (2017) suggest that masses of young disks derived from dust observations may be underestimated.

The more direct way to measure gas mass in disks is to observe molecular emission lines. Although disks mostly consist of molecular hydrogen and helium, these components either cannot be observed directly or trace only a limited disk region (see, e.g., Carmona et al. 2011), and other mass tracers are commonly employed, which require knowledge of a conversion factor from tracer mass to $\mathrm{H}_{2}$ mass. In this respect, hydrogen deuteride (HD) seems to be a good candidate (Bergin et al. 2013; McClure et al. 2016) as it is well coupled to molecular hydrogen and also has a relatively high abundance of $3 \cdot 10^{-5}$ relative to $\mathrm{H}_{2}$, although using it as a mass tracer may lead to a factor of 3 uncertainty (Trapman et al. 2017). Currently, the only facility that will be able to produce new HD measurements will be SOFIA with its forthcoming HIRMES spectrograph.

A widely used molecular mass tracer in disks is carbon monoxide (CO), as it is one of the most abundant species in interstellar and circumstellar material and possesses rotational transitions that are easy to observe. Hydrogen mass determination using CO lines is usually applied to diffuse clouds (Goldsmith et al. 1997; Bolatto et al. 2013), but now it is also commonly used for protoplanetary disks (Williams \& Best 2014; Ansdell et al. 2016; Miotello et al. 2016; Williams \& McPartland 2016). However, the CO-based disk mass determination method has some recognized problems, too. First, $\mathrm{CO}$ emission is optically thick, which can be circumvented by observing $\mathrm{CO}$ isotopologues ${ }^{13} \mathrm{CO}, \mathrm{C}^{18} \mathrm{O}, \mathrm{C}^{17} \mathrm{O}$, and also ${ }^{13} \mathrm{C}^{18} \mathrm{O}$, which has been recently observed in the TW Hya disk (Zhang et al. 2017). Their lines are typically optically thin as they are less abundant (Qi et al. 2011). The second problem is that $\mathrm{CO}$, along with its isotopologues, 
freezes out in cold dense regions of the disk midplane and becomes non-observable (Henning \& Semenov 2013).

Given the lack of good alternatives, CO observations are still vital for the determination of protoplanetary disk mass. However, its application for that purpose should be validated with theoretical models that include both radiation transfer (RT) and chemistry. Williams \& Best (2014) constructed a model of $\mathrm{CO}$ distribution over a selection of parameterized protoplanetary disks, assuming that $\mathrm{CO}$ is frozen in those regions of the disk where the temperature is below $20 \mathrm{~K}$ and is photodissociated above the vertical $\mathrm{H}_{2}$ column density of $1.3 \times 10^{21} \mathrm{~cm}^{-2}$. In the remaining part of the disk, the CO abundance relative to $\mathrm{H}_{2}$ was assumed to be equal to the socalled "interstellar" value, $10^{-4}$ (France et al. 2014). Williams $\&$ Best (2014) pointed out that under these assumptions, CO mass in this warm molecular zone represents a significant fraction of the total $\mathrm{CO}$ mass and thus traces the bulk of the disk gas mass. However, the CO-based disk gas masses derived so far seem to be systematically smaller than the masses derived from dust observations. ALMA disk surveys in Lupus, Chamaeleon, $\sigma$ Orionis, and other star-forming regions by Ansdell et al. (2016, 2017) based on the same theoretical model suggest very high dust-to-gas mass ratios up to $1 / 10$.

Williams \& Best (2014) suggested that the difference between the CO-based disk mass and the dust-based disk mass can be both real (reflecting low gas-to-dust mass ratios) and related to an uncertainty in the CO-to- $\mathrm{H}_{2}$ abundance ratio, if, for example, some other $\mathrm{CO}$ depletion pathways are more efficient. This possibility was explored by Miotello et al. (2016) with the isotope-selective chemical model by means of artificially reduced carbon abundance. However, they only considered simple hydrogenation surface processes, which may somewhat decrease gas-phase $\mathrm{CO}$ abundance. They noted that the uncertainty in the CO-based gas mass estimate can be reduced if some information on the disk structure is available. A continuation of this study was presented by Miotello et al. (2017), who confirmed that simplified surface chemistry may be the cause of discrepancy of disk masses estimated using CO and dust observations.

A more detailed surface chemistry model was utilized in the work of $\mathrm{Yu}$ et al. (2016). These authors considered CO evolution in the model of an accretion-heated disk and found that even inside a $\mathrm{CO}$ snowline, gas-phase $\mathrm{CO}$ abundance can be reduced significantly due to the conversion of $\mathrm{CO}$ into other less volatile species, like $\mathrm{CO}_{2}$ and complex organic molecules, which is a common effect at various stages of star formation (Vasyunina et al. 2012). However, only a limited number of disk models was considered in this study, and only the inner part of the disk (within $70 \mathrm{au}$ ) was analyzed. Also, Yu et al. (2016) assumed that significant dust evolution has already taken place in the disks, so that most dust grains have coagulated into larger particles, limiting the efficiency of both $\mathrm{CO}$ freeze-out and surface conversion into other molecules. $\mathrm{Yu}$ et al. (2017) extended this study and came to the conclusion that the chemical depletion of CO may lead to a significant underestimation of the disk gas masses.

In this study, we intend to combine a large grid of disk physical models with a comprehensive chemical model, which includes a detailed treatment of surface chemistry. We check the degree of reliability of $\mathrm{CO}$ as a gas mass tracer with realistic variations of the disk parameters and the uncertainties related to $\mathrm{CO}$ chemical depletion. We perform an analysis of the carbon partition into gas and solid species. The computed set of models is used to look for other possible tracers of disk gas mass.

\section{Model}

The basis for our study is a set of $\sim 1000$ disk models (described in detail in Section 2.3), which allows the distribution of gas-phase and surface molecular abundances for a selection of disk structural parameters to be studied. We utilize a modified version of the ANDES code (Akimkin et al. 2013) combined with the updated ALCHEMIC network (Semenov \& Wiebe 2011) to follow the evolution of molecular abundances.

\subsection{Physical Structure}

For the parameterization of the disk surface density, we adopt the commonly used tapered power-law profile (Hartmann et al. 1998)

$$
\Sigma(R)=\Sigma_{0}\left(\frac{R}{R_{\mathrm{c}}}\right)^{-\gamma} e^{-\left(R / R_{\mathrm{c}}\right)^{2-\gamma}}
$$

where $\Sigma_{0}$ in $\mathrm{g} \mathrm{cm}^{-2}$ is the surface density normalization, $\gamma$ parameterizes the radial dependence of the disk viscosity, and $R_{\mathrm{c}}$ is a characteristic radius of the disk. $\Sigma_{0}$ can be calculated from the total disk mass as

$$
\Sigma_{0}=\frac{M_{\mathrm{disk}}(2-\gamma)}{2 \pi R_{\mathrm{c}}^{2}} .
$$

So, the surface density radial profile depends on three parameters: $M_{\text {disk }}, \gamma$, and $R_{\mathrm{c}}$.

We assume that the disk is axially symmetric and its vertical structure at each radius is determined by the hydrostatic equilibrium

$$
\begin{gathered}
\frac{\partial P(R, z)}{\partial z}=-\rho(R, z) \frac{z G M_{\star}}{\left(R^{2}+z^{2}\right)^{3 / 2}}, \\
P=\frac{k_{\mathrm{B}} T(R, z)}{\mu m_{\mathrm{p}}} \rho(R, z),
\end{gathered}
$$

where the disk temperature distribution $T(R, z)$ is calculated using a simple parameterization (Williams \& Best 2014; Isella et al. 2016),

$$
T(R, z)= \begin{cases}T_{\mathrm{a}}\left(R, z=z_{\mathrm{q}}\right)+\left[T_{\mathrm{m}}(R)-T_{\mathrm{a}}\left(R, z=z_{\mathrm{q}}\right)\right]\left(\cos \frac{\pi z}{2 z_{\mathrm{q}}(R)}\right)^{2}, & \text { if }|z|<z_{\mathrm{q}}(R), \\ T_{\mathrm{a}}(R, z), & \text { if }|z| \geqslant z_{\mathrm{q}}(R) .\end{cases}
$$


Rather than adding the disk atmosphere and midplane temperatures $T_{\mathrm{m}}$ and $T_{\mathrm{a}}$ to our set of parameters, we calculate them at the base of the central luminosity source (star plus accretion region). The optically thin disk atmosphere can be easily ray-traced as dust thermal radiation does not contribute significantly to the source function. The dust temperature is determined from the thermal balance equation,

$$
\int_{0}^{\infty} \kappa_{\nu} J_{\nu} d \nu=\int_{0}^{\infty} \kappa_{\nu} B_{\nu}\left(T_{\mathrm{a}}\right) d \nu
$$

where $\kappa_{\nu}$ in $\mathrm{cm}^{-1}$ is the dust absorption coefficient and $J_{\nu}$ is the mean intensity of the radiation coming directly from the central star, accretion region, and interstellar medium,

$$
\begin{aligned}
J_{\nu}= & J_{\nu, \mathrm{s}}+J_{\nu, \mathrm{acc}}+J_{\nu, \mathrm{ISRF}}=W_{\star} B_{\nu}\left(T_{\star}\right) e^{-\tau_{\nu, 1}} \\
& +W_{\mathrm{acc}} B_{\nu}\left(T_{\mathrm{acc}}\right) e^{-\tau_{\nu, 1}}+\frac{1}{3} I_{\mathrm{ISRF}}\left(e^{-\tau_{\nu, 2}}\right. \\
& \left.+e^{-\tau_{\nu, 3}}+e^{-\tau_{\nu, 4}}\right) .
\end{aligned}
$$

Here, $\quad W_{\star}=\frac{1}{4} R_{\star}^{2} /\left(R^{2}+z^{2}\right) \quad$ and $\quad W_{\mathrm{acc}}=\frac{1}{4} R_{\mathrm{acc}}^{2} /\left(R^{2}+z^{2}\right)$ represent the dilution factors for the radiation from the central star and accretion region, and $R_{\star}$ and $T_{\star}$ are the stellar radius and effective temperature. The accretion luminosity is simulated by the additional central source of irradiation with effective radius $R_{\text {acc }}$ and effective temperature $T_{\text {acc }}$, so that $L_{\mathrm{acc}}=4 \pi R_{\mathrm{acc}}^{2} \sigma_{\mathrm{SB}} T_{\mathrm{acc}}^{4}=(3 / 2) G M_{\star} \dot{M} / R_{\star}$. We assume a typical accretion rate of $\dot{M}=10^{-8} M_{\odot} \mathrm{yr}^{-1}$ and $T_{\text {acc }}=15,000 \mathrm{~K}$ to calculate the effective radius of an accretion region, $R_{\text {acc }}$. The interstellar radiation field is adopted from Mathis et al. (1983).

The optical depth in Equation (7), $\tau_{\nu, k}=\int\left(\kappa_{\nu}+\sigma_{\nu}\right) d l$, is calculated along four directions: to and from the $\operatorname{star}(k=1,2)$, and up and down $(k=3,4)$. Dust optical properties are computed from the Mie theory for astrosilicate grains (Laor \& Draine 1993) assuming a dust-to-gas ratio of 0.01. We use a spatial grid $R_{n}$ and $z_{n m}$ such that $z_{n m} / R_{n}$ is constant for a given $m$. This greatly reduces the computational cost for the raytracing procedure as all spatial points with a fixed mindex are located on the same line.

We define $z_{\mathrm{q}}(R)$ as the height at which $T_{\mathrm{a}}$ has a maximum at a given $R$. Above $z_{\mathrm{q}}(R)$, the dust temperature decreases with $z$ due to increasing distance from the star; the drop in temperature below $z_{\mathrm{q}}(R)$ is explained by the absorption. This also means that the source function starts to be dominated by the dust thermal infrared radiation and not by the radiation from the star. In this regime, the approximation for radiation intensity (7) is no longer valid. The temperature profile below $z_{\mathrm{q}}(R)$ is set according to Equation (5), with the midplane temperature adopted from the optically thick case of the two-layered model (Chiang \& Goldreich 1997; Dullemond et al. 2001),

$$
T_{\mathrm{m}}^{4}(R)=\frac{1}{2} \varphi\left[T_{\star}^{4}\left(\frac{R_{\star}}{R}\right)^{2}+T_{\mathrm{acc}}^{4}\left(\frac{R_{\mathrm{acc}}}{R}\right)^{2}\right],
$$

where $\varphi=0.05$ is a flaring angle (Dullemond \& Dominik 2004), and the factor $\frac{1}{2}$ reflects the fact that only half of the infrared radiation from the super-heated upper layer of the disk heats the midplane and the other half is emitted outward. This midplane temperature differs from the disk effective temperature by a factor of $\sqrt[4]{2}$. Gas and dust temperatures are assumed to be equal. We use iterations to obtain self-consistent density and temperature structures, which is important for the ray- tracing procedure and calculation of the atmosphere temperature. However, in the calculation of the midplane temperature, we keep the flaring angle fixed to avoid disk self-shadowing as this effect must be studied by means of a more sophisticated model. The adopted model for the disk physical structure enables us to perform astrochemical simulations for a large set of models while keeping the adequate level of accuracy.

The values of the stellar radius $R_{\star}$, effective temperature $T_{\star}$, and stellar mass $M_{\star}$ used in the above parameterizations are not independent. We take $M_{\star}$ as a primary parameter and calculate $R_{\star}$ and $T_{\star}$ from the evolutionary tracks of Baraffe et al. (2015), assuming a stellar age of $3 \mathrm{Myr}$. As their model is restricted to low-mass stars, we only consider T Tauri-type stars with masses between 0.5 and $1.4 M_{\odot}$. Stellar and accretion luminosities are assumed to be constant during the chemical run. However, luminosity bursts may have a lasting impact on the disk chemical structure and are a topic of current research (Harsono et al. 2015; Rab et al. 2017).

Overall, we have four parameters, $M_{\mathrm{disk}}, \gamma, R_{\mathrm{c}}$, and $M_{\star}$, that determine the physical structure of the disk. The spatial grid has 50 points in the radial direction between 1 and $1000 \mathrm{au}$, and 80 points in the vertical direction from the midplane to $z / R=0.5$.

\subsection{Chemical Model}

The chemical structure is calculated with the modified version of the thermochemical non-equilibrium ANDES code (Akimkin et al. 2013). The system of chemical kinetics equations was updated to account for the gas-grain chemistry of the ALCHEMIC model (Semenov \& Wiebe 2011). The gasphase part was benchmarked with the NAHOON code (Wakelam et al. 2015), which resulted in fixing several noncritical bugs. Some rate coefficients were updated in accordance with the KIDA14 database. Overall, the chemical network contains 650 species involved in 7807 reactions. No isotope-selective chemistry is considered.

The original version of the ANDES code contains a module to calculate the thermal balance of gas and dust. We replaced this module in the current study by a phenomenological setup for the thermal structure in order to reduce computational costs for modeling a large set of disks. However, the 2D RT of UV radiation was implemented in order to calculate photoreaction rates. The RT is based on the short characteristics method and allows for the radiation field calculation up to optical depths $\tau_{\mathrm{UV}} \sim 10$. The MRN grain size distribution (Mathis et al. 1977) and dust-to-gas ratio of 0.01 are taken as the dust model for the RT modeling in the disk atmosphere. We calculate RT for the stellar and interstellar radiation field and also consider X-rays, cosmic rays (CR), and radioactive nuclides as sources of ionization. The spectrum of a central star is assumed to be a blackbody with an effective temperature corresponding to a given stellar mass plus a UV excess produced by accretion (as stated above). Although the real stellar spectra may not be wellrepresented by a blackbody, in the context of our study, only the relation between the visible and far-UV spectrum parts is important. The X-ray ionization rate is calculated on the basis of the Bai \& Goodman (2009) model (their Equation (21)), assuming X-ray luminosity $L_{\mathrm{X}}=10^{30} \mathrm{erg} \mathrm{s}^{-1}$ and $T_{\mathrm{X}}=3 \mathrm{keV}$. We use $\zeta_{0}=1.3 \times 10^{-17} \mathrm{~s}^{-1}$ for the unattenuated cosmic-ray ionization rate and an attenuation length of $96 \mathrm{~g} \mathrm{~cm}^{-2}$ (Sano 
Table 1

Initial Abundances (Relative to $\mathrm{H}$ Nuclei)

\begin{tabular}{|c|c|c|c|c|c|c|c|}
\hline Species & $\overline{\mathrm{H}} \mathrm{H}_{2}$ & $\mathrm{H}$ & $\mathrm{He}$ & $\overline{\mathrm{C}^{+}}$ & $\mathrm{N}$ & $\overline{\mathrm{O}}$ & $\mathrm{S}^{+}$ \\
\hline Abundance & 0.499 & 0.002 & 0.09 & $7.3 \cdot 10^{-5}$ & $2.14 \cdot 10^{-5}$ & $1.76 \cdot 10^{-4}$ & $8 \cdot 10^{-8}$ \\
\hline Species & $\mathrm{Si}^{+}$ & $\mathrm{Fe}^{+}$ & $\mathrm{Na}^{+}$ & $\mathrm{Mg}^{+}$ & $\mathrm{P}^{+}$ & $\mathrm{Cl}^{+}$ & $\ldots$ \\
\hline Abundance & $8 \cdot 10^{-9}$ & $3 \cdot 10^{-9}$ & $2 \cdot 10^{-9}$ & $7 \cdot 10^{-9}$ & $2 \cdot 10^{-10}$ & $1 \cdot 10^{-9}$ & $\ldots$ \\
\hline
\end{tabular}

et al. 2000) and $\zeta_{\mathrm{RA}}=6.5 \times 10^{-19} \mathrm{~s}^{-1}$ for the ionization rate by radioactive elements.

The initial abundances are presented in Table 1 and correspond to the "low-metals" case of Lee et al. (1998), unless otherwise noted.

Apart from gas-phase processes, our chemical network includes surface reactions along with accretion and desorption. As many species tend to freeze out onto dust particles, which makes them unobservable, surface chemistry is crucial for such models. We adopt the ratio of diffusion energy to desorption energy of 0.5 and account for tunneling through reaction barriers. Single-layer surface chemistry is considered. Our treatment of surface chemistry includes reactive desorption with an efficiency of $1 \%$, which means that in two-body surface reactions, $99 \%$ of the product species stay on the surface and $1 \%$ is released into gas, although a more detailed treatment of reactive desorption is already available in some astrochemical models (Vasyunin et al. 2017). The representative size of dust grains in the grain chemistry treatment is assumed to be $0.1 \mu \mathrm{m}$. In protoplanetary disks, dust is supposed to grow, which reduces the available dust surface and slows down the surface chemistry. Also, grown dust makes the disk more transparent to stellar radiation. However, the theoretical description of dust evolution is a complicated task, especially when taking into account such poorly studied effects such as grain charging (Okuzumi 2009; Akimkin 2015; Ivlev et al. 2016). Therefore, we ignore dust evolution effects and focus on varying the disk macrophysical parameters.

\subsection{Set of Models}

Like Williams \& Best (2014), we create a grid of models with variable parameters responsible for the disk's physical structure. As we already mentioned, these parameters are the disk mass $M_{\text {disk }}$, stellar mass $M_{\star}$, effective radius of the disk $R_{\mathrm{c}}$, and power-law index $\gamma$. We vary them within bounds typical for protoplanetary disks around T Tauri stars. Specific ranges for each parameter are presented in Table 2.

We calculate the 2D distributions of 650 species for time moments of $0.5,1$, and $3 \mathrm{Myr}$, and use these distributions to compute the total species masses in the disk. In Section 3.1, we employ a random set of models, with every parameter being uniformly distributed inside the ranges (for the disk mass, it is $\log _{10} M_{\text {disk }}$ that is distributed uniformly). In Section 3.3, we use a grid of models with fixed nodes and present various cuts through the parameter space.

\section{Results}

\subsection{Potential Gas Mass Tracers}

The list of molecules observed in protoplanetary disks is currently not very extensive and includes $\mathrm{CN}, \mathrm{HCN}, \mathrm{HNC}, \mathrm{CS}$, $\mathrm{SO}, \mathrm{H}_{2} \mathrm{CO}, \mathrm{CCH}, \mathrm{HC}_{3} \mathrm{~N}, \mathrm{CH}_{3} \mathrm{CN}, \mathrm{C}_{3} \mathrm{H}_{2}, \mathrm{C}_{2} \mathrm{H}_{2}, \mathrm{OH}, \mathrm{HCO}^{+}$, $\mathrm{N}_{2} \mathrm{H}^{+}, \mathrm{CH}^{+}, \mathrm{C}^{+}, \mathrm{O}, \mathrm{NH}_{3}$, and $\mathrm{CH}_{3} \mathrm{OH}$ (Dutrey et al. 1997,
Table 2

Range of Parameters Used in the Set of Models

\begin{tabular}{ll}
\hline \hline Parameter & Value \\
\hline$M_{\text {disk }}$ & $10^{-4} \div 10^{-1} M_{\odot}$ \\
$M_{\star}$ & $0.5 \div 1.4 M_{\odot}$ \\
$R_{\mathrm{c}}$ & $30 \div 200 \mathrm{au}$ \\
$\gamma$ & $0.5 \div 1.5$ \\
\hline
\end{tabular}

2011; Thi et al. 2011; Chapillon et al. 2012; Qi et al. 2013; Öberg et al. 2015; Guilloteau et al. 2016; Salinas et al. 2016; Walsh et al. 2016). It would be interesting to find out if some of them could possibly serve as a gas mass tracer alternative to commonly used CO. Also, pending future discoveries of molecules in protoplanetary disks, we do not exclude from consideration other chemical species present in the model.

The total disk abundance of a prospective disk mass tracer $X_{i}=N_{i} / N_{\langle\mathrm{H}\rangle}$ should be correlated with the disk mass, while being less dependent on other physical properties of the disk, such as the density profile, disk radius, or properties of the central star. The correlation should not necessarily be linear, implying the independence of the total abundance from the disk mass. In principle, the species abundance can be any monotonic function of the disk mass. Also, it should not vary significantly with time.

To assess the potential applicability of the species as a disk mass tracer, we need to introduce some quantitative gauges. For every $j$ th disk model in the ensemble, we calculate the total logarithmic abundance of the $i$ th species, $x_{i j}=\log _{10}\left(X_{i j}\right)$. The value $\overline{x_{i}}$, averaged over the disk model ensemble, represents a typical (logarithm of) total disk abundance of the $i$ th species. We employ its dispersion $s_{i}^{2}=\overline{\left(x_{i j}-\overline{x_{i}}\right)^{2}}$ as a simple quantitative measure of suitability of the $i$ th species as a mass tracer. Further, we refer to the standard deviation $s_{i}$ as a "scatter parameter" to stress the limitation of the approach. First, the distribution of the protoplanetary disk parameters is not wellknown and quite probably differs from the uniform distribution assumed during the disk ensemble generation. Second, the scatter may not reflect the nonlinear scaling between the disk and species mass. However, generally speaking, the smaller the scatter, the better the species performs as a mass tracer.

Table 3 summarizes this simple statistical analysis and contains the total disk abundances and scatter parameters for the model age of $3 \mathrm{Myr}$, sorted by increasing $s_{i}$. For other chemical ages, the values of the scatter parameter are about the same, slightly growing with time. We include components with average total disk abundance greater than $10^{-11}$, excluding ices and common species like $\mathrm{H}_{2}$ and $\mathrm{He}$. All of the detected species listed above are present in the table as well. The species that are potentially observable with JWST but not with ALMA are marked with a star $\left(^{\star}\right)$. Total disk abundances for ice species are given in the Appendix in the table analogous to Table 3. 
Table 3

Average Total Disk Abundance $\overline{X_{i}}=10^{\overline{x_{i}}}$ and Scatter $s_{i}$ for Selected Species

\begin{tabular}{|c|c|c|c|c|c|c|c|c|}
\hline Species & $\overline{X_{i}}$ & $s_{i}(\operatorname{dex})$ & Species & $\overline{X_{i}}$ & $s_{i}(\operatorname{dex})$ & Species & $\overline{X_{i}}$ & $s_{i}(\operatorname{dex})$ \\
\hline $\mathrm{N}_{2}$ & $2.0 \times 10^{-6}$ & 0.25 & $\mathrm{C}_{6}$ & $1.5 \times 10^{-11}$ & 0.45 & $\mathrm{C}$ & $4.9 \times 10^{-6}$ & 0.64 \\
\hline $\mathrm{NH}_{3}$ & $3.2 \times 10^{-9}$ & 0.27 & $\mathrm{HCN}$ & $2.4 \times 10^{-9}$ & 0.45 & $\mathrm{O}_{3}$ & $5.5 \times 10^{-8}$ & 0.64 \\
\hline $\mathrm{CH}_{3}$ & $3.6 \times 10^{-10}$ & 0.28 & $\mathrm{Si}$ & $1.7 \times 10^{-10}$ & 0.46 & $\mathrm{O}$ & $2.1 \times 10^{-5}$ & 0.66 \\
\hline $\mathrm{CO}$ & $8.0 \times 10^{-6}$ & 0.28 & $\mathrm{C}_{4} \mathrm{H}$ & $1.0 \times 10^{-10}$ & 0.46 & $\mathrm{C}_{2} \mathrm{H}_{6}$ & $2.3 \times 10^{-9}$ & 0.66 \\
\hline $\mathrm{H}_{3}^{+}$ & $2.2 \times 10^{-10}$ & 0.28 & ${ }^{\star} \mathrm{C}_{2} \mathrm{H}_{2}$ & $4.3 \times 10^{-10}$ & 0.47 & $\mathrm{CH}_{3}^{+}$ & $3.8 \times 10^{-11}$ & 0.73 \\
\hline${ }^{\star} \mathrm{CO}_{2}$ & $5.2 \times 10^{-7}$ & 0.28 & $\mathrm{Na}$ & $1.9 \times 10^{-10}$ & 0.48 & $\mathrm{C}_{2} \mathrm{H}$ & $6.6 \times 10^{-10}$ & 0.74 \\
\hline $\mathrm{H}_{2} \mathrm{CO}$ & $1.3 \times 10^{-10}$ & 0.29 & $\mathrm{~N}_{2} \mathrm{H}^{+}$ & $1.3 \times 10^{-11}$ & 0.48 & $\mathrm{Na}^{+}$ & $1.8 \times 10^{-10}$ & 0.74 \\
\hline $\mathrm{H}_{3} \mathrm{O}^{+}$ & $2.6 \times 10^{-10}$ & 0.30 & $\mathrm{CS}$ & $4.7 \times 10^{-10}$ & 0.49 & $\mathrm{~N}$ & $1.5 \times 10^{-6}$ & 0.75 \\
\hline $\mathrm{H}_{2} \mathrm{CN}^{+}$ & $1.6 \times 10^{-11}$ & 0.31 & $\mathrm{Mg}$ & $4.0 \times 10^{-11}$ & 0.49 & $\mathrm{H}_{5} \mathrm{C}_{3} \mathrm{~N}$ & $2.2 \times 10^{-11}$ & 0.77 \\
\hline $\mathrm{H}_{2} \mathrm{CS}$ & $1.6 \times 10^{-11}$ & 0.32 & $\mathrm{H}_{2} \mathrm{C}_{3} \mathrm{O}$ & $4.7 \times 10^{-10}$ & 0.50 & $\mathrm{CH}_{2}$ & $5.6 \times 10^{-10}$ & 0.77 \\
\hline $\mathrm{NH}_{2}$ & $3.5 \times 10^{-10}$ & 0.32 & $\mathrm{MgH}_{2}$ & $5.2 \times 10^{-10}$ & 0.50 & $\mathrm{HC}_{2} \mathrm{O}$ & $6.9 \times 10^{-11}$ & 0.80 \\
\hline $\mathrm{SO}_{2}$ & $2.1 \times 10^{-10}$ & 0.32 & $\mathrm{HS}$ & $2.1 \times 10^{-10}$ & 0.51 & $\mathrm{~N}_{2} \mathrm{O}$ & $1.2 \times 10^{-10}$ & 0.81 \\
\hline HNO & $1.4 \times 10^{-10}$ & 0.33 & $\mathrm{CH}_{2} \mathrm{CO}$ & $1.8 \times 10^{-10}$ & 0.51 & $\mathrm{~S}^{+}$ & $4.9 \times 10^{-9}$ & 0.81 \\
\hline $\mathrm{C}_{3}$ & $1.3 \times 10^{-8}$ & 0.34 & $\mathrm{CH}_{5} \mathrm{~N}$ & $2.5 \times 10^{-11}$ & 0.51 & $\mathrm{HCCN}$ & $5.0 \times 10^{-11}$ & 0.81 \\
\hline $\mathrm{C}_{5} \mathrm{H}_{3}$ & $2.5 \times 10^{-11}$ & 0.35 & ${ }^{\star} \mathrm{H}_{2} \mathrm{O}$ & $4.0 \times 10^{-7}$ & 0.51 & $\mathrm{P}^{+}$ & $1.1 \times 10^{-11}$ & 0.82 \\
\hline SO & $2.7 \times 10^{-9}$ & 0.37 & $\mathrm{Cl}$ & $1.8 \times 10^{-10}$ & 0.51 & $\mathrm{Si}^{+}$ & $5.1 \times 10^{-10}$ & 0.83 \\
\hline $\mathrm{C}_{3} \mathrm{H}$ & $1.1 \times 10^{-10}$ & 0.39 & $\mathrm{CH}_{3} \mathrm{CN}$ & $2.0 \times 10^{-11}$ & 0.52 & $\mathrm{CH}$ & $1.5 \times 10^{-9}$ & 0.83 \\
\hline $\mathrm{OCN}$ & $1.7 \times 10^{-10}$ & 0.40 & $\mathrm{HNC}$ & $1.2 \times 10^{-9}$ & 0.52 & $\mathrm{Fe}^{+}$ & $1.6 \times 10^{-10}$ & 0.84 \\
\hline${ }^{\star} \mathrm{OH}$ & $1.4 \times 10^{-8}$ & 0.41 & $\mathrm{H}_{2} \mathrm{O}_{2}$ & $4.0 \times 10^{-9}$ & 0.53 & $\mathrm{Mg}^{+}$ & $3.3 \times 10^{-10}$ & 0.84 \\
\hline $\mathrm{P}$ & $1.5 \times 10^{-11}$ & 0.41 & $\mathrm{C}_{4} \mathrm{H}_{2}$ & $2.4 \times 10^{-11}$ & 0.53 & $\mathrm{O}_{2} \mathrm{H}$ & $1.3 \times 10^{-11}$ & 0.85 \\
\hline $\mathrm{HC}_{3} \mathrm{O}$ & $3.2 \times 10^{-11}$ & 0.41 & $\mathrm{C}_{2} \mathrm{H}_{4}$ & $1.7 \times 10^{-11}$ & 0.54 & $\mathrm{CN}$ & $1.4 \times 10^{-9}$ & 0.86 \\
\hline $\mathrm{H}_{2} \mathrm{~S}$ & $1.3 \times 10^{-9}$ & 0.41 & $\mathrm{C}_{5}$ & $1.1 \times 10^{-10}$ & 0.54 & $\mathrm{HC}_{3} \mathrm{~N}$ & $1.6 \times 10^{-11}$ & 0.86 \\
\hline $\mathrm{FeH}$ & $2.4 \times 10^{-10}$ & 0.42 & $\mathrm{C}_{3} \mathrm{H}_{2}$ & $8.7 \times 10^{-11}$ & 0.54 & $\mathrm{C}^{+}$ & $1.5 \times 10^{-6}$ & 0.88 \\
\hline $\mathrm{C}_{4}$ & $1.6 \times 10^{-10}$ & 0.43 & $\mathrm{C}_{5} \mathrm{H}_{2}$ & $2.1 \times 10^{-11}$ & 0.55 & $\mathrm{C}_{9} \mathrm{H}_{2}$ & $2.3 \times 10^{-11}$ & 0.94 \\
\hline NO & $4.1 \times 10^{-9}$ & 0.43 & ${ }^{\star} \mathrm{CH}_{4}$ & $7.7 \times 10^{-8}$ & 0.55 & $\mathrm{C}_{5} \mathrm{H}_{4}$ & $2.9 \times 10^{-11}$ & 0.97 \\
\hline $\mathrm{NH}$ & $9.6 \times 10^{-11}$ & 0.43 & $\mathrm{CH}_{3} \mathrm{OH}$ & $9.3 \times 10^{-11}$ & 0.56 & $\mathrm{C}_{2}$ & $1.2 \times 10^{-9}$ & 1.02 \\
\hline S & $6.5 \times 10^{-9}$ & 0.43 & $\mathrm{C}_{5} \mathrm{H}$ & $2.2 \times 10^{-11}$ & 0.57 & $\mathrm{CH}_{2} \mathrm{NH}_{2}$ & $4.8 \times 10^{-11}$ & 1.09 \\
\hline $\mathrm{SiO}$ & $3.4 \times 10^{-10}$ & 0.43 & $\mathrm{CH}_{2} \mathrm{OH}$ & $4.1 \times 10^{-11}$ & 0.59 & $\mathrm{CH}_{3} \mathrm{NH}$ & $4.7 \times 10^{-11}$ & 1.09 \\
\hline${ }^{\star} \mathrm{O}_{2}$ & $2.4 \times 10^{-6}$ & 0.44 & $\mathrm{HCO}^{+}$ & $8.7 \times 10^{-11}$ & 0.60 & $\mathrm{CH}^{+}$ & $3.7 \times 10^{-13}$ & 1.19 \\
\hline $\mathrm{HNCO}$ & $1.9 \times 10^{-9}$ & 0.44 & $\mathrm{HCOOH}$ & $1.3 \times 10^{-11}$ & 0.63 & $\mathrm{~N}_{2} \mathrm{H}_{2}$ & $6.9 \times 10^{-11}$ & 1.22 \\
\hline
\end{tabular}

The top of the table is occupied by $\mathrm{N}_{2}, \mathrm{NH}_{3}, \mathrm{CH}_{3}, \mathrm{CO}, \mathrm{H}_{3}^{+}$, and $\mathrm{CO}_{2}$, and in Figure 1, their total masses are shown as functions of the total disk mass. In each panel, a solid line is shown with the slope corresponding to the linear scaling of the species mass with the disk mass at the average total abundance $\overline{X_{i}}=10^{\overline{x_{i}}}$ from Table 3. A scatter parameter is also indicated in each panel. Molecular nitrogen seems to be the best theoretical mass tracer as its mass scales almost linearly with the disk mass over the entire range of considered disk masses. This molecule, like $\mathrm{H}_{2}$, has no rotational transitions and thus cannot be observed directly, but ${ }^{14} \mathrm{~N}^{15} \mathrm{~N}$ has such transitions. Despite the fact that the mass difference between ${ }^{14} \mathrm{~N}$ and ${ }^{15} \mathrm{~N}$ is small and hence the dipole moment is very weak, theoretically, one could detect its fundamental rotational line at $4.34 \mu \mathrm{m}$ in disks.

The CO total mass also shows nearly linear scaling with the disk mass, but with a scatter that increases somewhat in more massive disks. Disks with lower $\gamma$ values (shallower density profiles) tend to have smaller $\mathrm{CO}$ abundance, while more compact disks (with lower $\gamma$ and $R_{\mathrm{c}}$, i.e., smaller and redder dots in Figure 1) are more $\mathrm{CO}$ rich. The reason is that in a compact disk, most mass is located in a warm disk area, where $\mathrm{CO}$ freeze-out is prohibited. As we consider larger and/or shallower disks, more mass is shifted to colder disk areas.

The dashed line in the top-left panel of Figure 1 indicates the maximum possible $\mathrm{CO}$ mass, corresponding to the assumption that all carbon atoms are locked in gas-phase CO. In this extreme case, $M_{\mathrm{CO}}=1.5 \times 10^{-3} M_{\text {disk }}$. According to our results, the computed total $\mathrm{CO}$ mass is much lower in all of the considered models, and its typical value is only $1.7 \times 10^{-4} M_{\text {disk }}$. Most carbon atoms are locked up not in
$\mathrm{CO}$ but in $\mathrm{CO}_{2}$ ice. Carbon redistribution is discussed in more detail in Section 3.2.

The scatter parameters for $\mathrm{NH}_{3}$ and $\mathrm{CH}_{3}$ are formally somewhat lower than the one for $\mathrm{CO}$. However, inspection of Figure 1 shows that the masses of these species depend more strongly on the disk structure than on the CO mass, if the disk mass exceeds $\sim 10^{-3} M_{\odot}$. Also, ammonia masses lose the linear scaling with disk masses in the least massive disks. Additionally, abundances of $\mathrm{NH}_{3}$ and $\mathrm{CH}_{3}$, as well as that of $\mathrm{H}_{3}^{+}$, are quite low, which hampers their detection. While $\mathrm{NH}_{3}$ has nevertheless been detected by Herschel in the nearby TW Hya disk (Salinas et al. 2016), the methyl radical and $\mathrm{H}_{3}^{+}$ have no allowed dipole moment as they are symmetric and planar. Potentially, $\mathrm{H}_{3}^{+}$can be traced by $\mathrm{o}-\mathrm{H}_{2} \mathrm{D}^{+}$; thus, even though we do not consider isotopic chemistry, $\mathrm{o}-\mathrm{H}_{2} \mathrm{D}^{+}$may also correlate well with the disk gas mass. However, detecting this species in disks is challenging even for ALMA (Chapillon et al. 2011).

Carbon dioxide looks quite promising. Its scatter only slightly exceeds that of $\mathrm{CO}$, and it will also be observable by JWST. Although the dependence of its mass on the disk mass is nonlinear in disks with the lowest masses, this is compensated by uniformly low scatter and linear scaling for disks with masses above $\sim 10^{-3} M_{\odot}$.

Overall, we conclude that $\mathrm{CO}$ is currently the best mass tracer, combining ease of detection and predictability of behavior. Among other species, some are better than others, with the scatter comparable to that of $\mathrm{CO}$ (see Figure 2). Water mass scales with the disk mass for $M_{\text {disk }}>10^{-3} M_{\odot}$, but in the least massive disks, the water abundance shows a significant 


\section{disk characteristic radius, au}

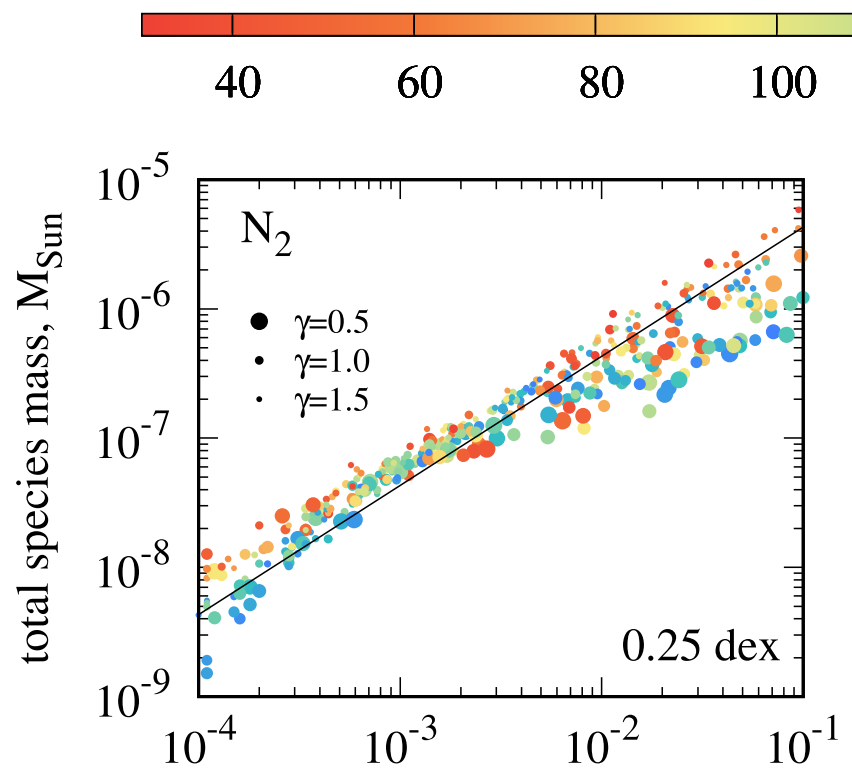

$\begin{array}{lllll}120 & 140 & 160 & 180 & 200\end{array}$
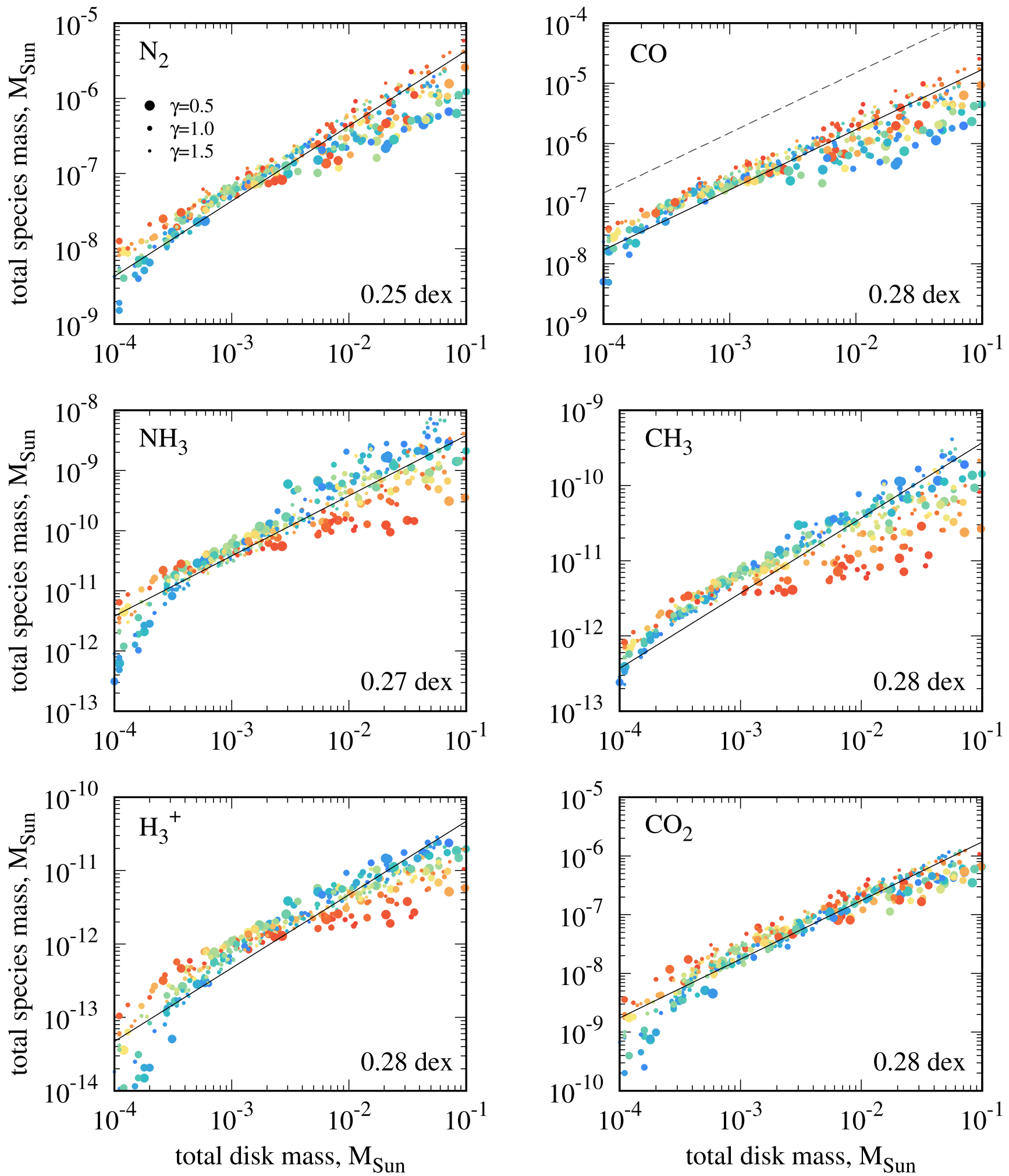

Figure 1. Total mass of species with the lowest scatter values in the disk as a function of the disk mass at an age of 3 Myr. The point size depicts the $\gamma$ value (larger symbols represent shallower surface density profiles), the point color represents the disk characteristic radius $R_{\mathrm{c}}$. The solid lines indicate linear scaling, with the slope equal to the average mass fraction. The dashed line corresponds to the $\mathrm{CO}$ amount if it were to contain all of the available carbon atoms. The scatter parameter is shown in the bottom-right corner of each panel. 
disk characteristic radius, au

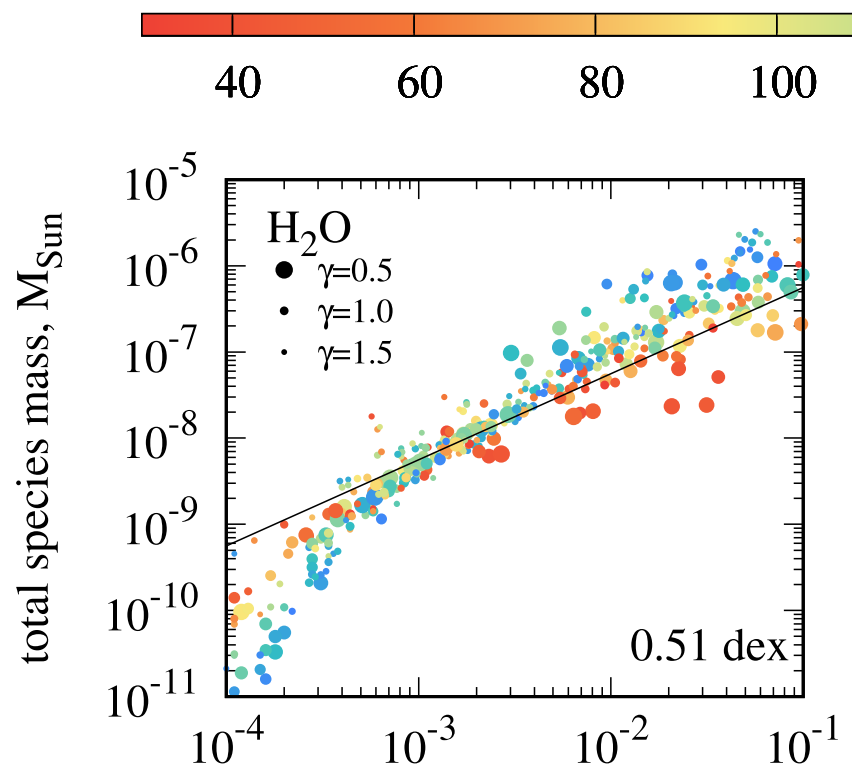

$\begin{array}{lllll}120 & 140 & 160 & 180 & 200\end{array}$
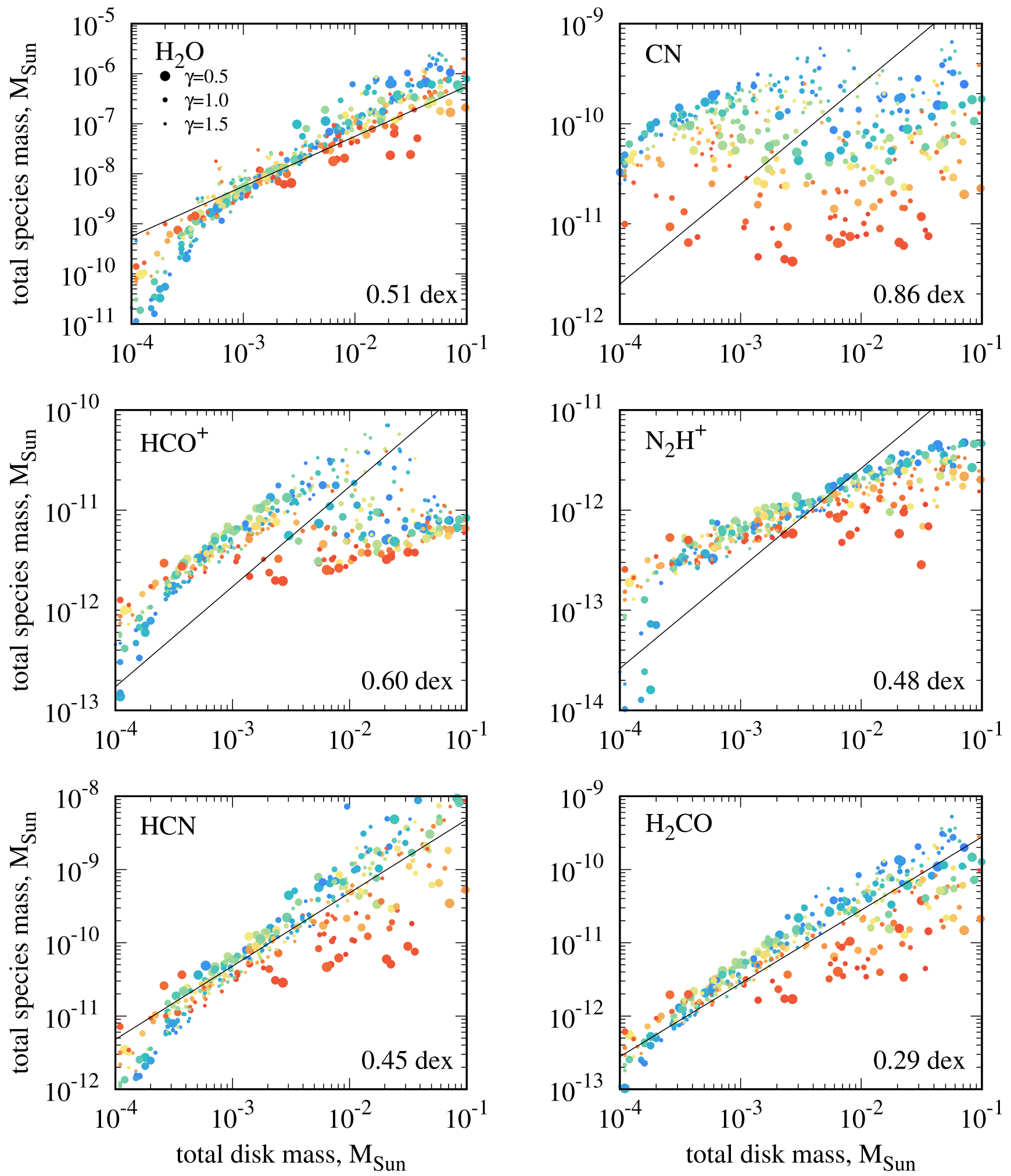

Figure 2. Same as in Figure 1, but for species with higher scatter values.

scatter (of about two orders of magnitude), with smaller $\gamma$ values and more extended disks corresponding to lower water masses. This seems to be interesting in view of the diverse results of Herschel water observations in the disks of DM Tau (Bergin et al. 2010) and TW Hya (Hogerheijde et al. 2011). Quite different water abundances 


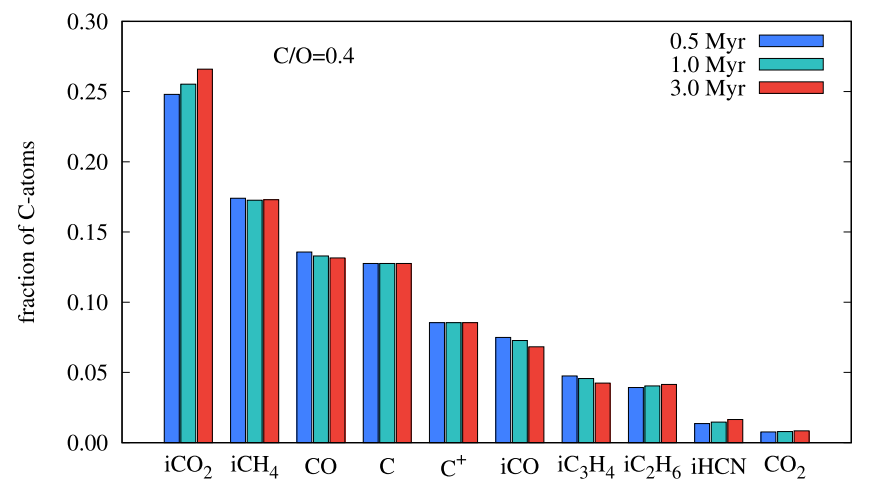

Figure 3. Distribution of carbon between major carbon-bearing molecules for different disk ages, averaged over the models. Ice species are denoted by a leading "i." $\mathrm{C} / \mathrm{O}=0.4$ is the ratio of carbon to oxygen elemental abundances.

may reflect not only evolutionary changes but also structural variations.

Other carbon-bearing species show a significant scatter across the entire disk mass range, and the scatter generally increases as the disk mass increases. But in some cases, the uncertainty can be reduced significantly if the disk size is known. Intriguingly, the $\mathrm{H}_{2} \mathrm{CO}$ emission detected in disks could potentially be used as a proxy of disk mass, since the $\mathrm{H}_{2} \mathrm{CO}$ disk mass factor has a low scatter similar to $\mathrm{CO}$ and is quite tight (albeit nonlinear), if most compact disks are excluded. On the other hand, the $\mathrm{H}_{2} \mathrm{CO}$ emission in disks shows a peculiar ring-like structure (Henning \& Semenov 2008; Loomis et al. 2015; Öberg et al. 2017), which makes such an analysis a difficult endeavor. The $\mathrm{CN}$ and $\mathrm{HCO}^{+}$scaling are sufficiently nonlinear, which hampers their use as mass tracers.

An interesting case is represented by $\mathrm{N}_{2} \mathrm{H}^{+}$. The dependence of its mass on the disk mass is quite tight over the entire $M_{\text {disk }}$ range and shows extreme sensitivity to the disk mass for $M_{\text {disk }}<3 \times 10^{-4} M_{\odot}$. However, at higher $M_{\text {disk }}$ values, the dependence of $\mathrm{N}_{2} \mathrm{H}^{+}$mass on the disk mass is much weaker. This should be related to $\mathrm{CO}$ behavior. In extremely low-mass disks, $\mathrm{CO}$ is depleted from the gas phase only due to freeze-out. In more massive disks, $\mathrm{CO}$ also experiences chemical depletion due to carbon redistribution into other species. The steeper left part of the $\mathrm{N}_{2} \mathrm{H}^{+}$graph (see Figure 2) reflects the developing chemical depletion of $\mathrm{CO}$, while at the shallower right part, this process is saturated. The change of $\mathrm{N}_{2} \mathrm{H}^{+}$abundance in more massive disks is explained by the shift of $\mathrm{N}_{2} \mathrm{H}^{+}$from the midplane to the upper layers.

\subsection{Carbon Redistribution}

In this section, we consider major carbon-bearing species and demonstrate that gaseous $\mathrm{CO}$ is not the main reservoir of carbon in the considered protoplanetary disks. For each disk, we find molecules that are major carbon reservoirs and calculate what percentage of carbon they comprise. The distribution of carbon among the top 10 carbon-bearing species is plotted in Figure 3. Three time moments are presented, $0.5,1$, and $3 \mathrm{Myr}$, showing that this distribution does not change much with the disk age.

There are two reasons for such a behavior. First, we show the carbon fraction integrated over the entire disk, and most of material contributing to this value is relatively warm and dense, allowing chemical equilibrium to be reached within $0.5 \mathrm{Myr}$. Second, the ice species shown are relatively simple, and their formation does not involve long-scale surface chemistry, so that they also have short typical chemical timescales.

We do see, however, some temporal changes in the carbon fraction for some species. The fraction of $\mathrm{CO}_{2}$ ice gradually increases with time, while fractions of $\mathrm{CO}$ in gas and ice diminish mostly due to the conversion of $\mathrm{iCO}$ into $\mathrm{iCO}_{2}$ on the dust surfaces. Other molecule fractions also demonstrate some trends, so the redistribution of carbon in disks slowly continues during the considered time.

The diagram in Figure 3 shows that carbon is mostly locked in $\mathrm{CO}_{2}$ ice $(27 \% \pm 8 \%)$ at $3 \mathrm{Myr}$, but a sufficient fraction of carbon stays in the elementary forms of $\mathrm{C}$ or $\mathrm{C}^{+}$. Among the 10 major carbon-bearing molecules, which constitute $95 \%$ of all available $\mathrm{C}$ atoms, only four are in the gas phase. The most abundant gas-phase species is $\mathrm{CO}$ with an average of $13 \%$, while gas-phase $\mathrm{CO}_{2}$ comprises only about $1 \%$. In other words, only every seventh $\mathrm{C}$ atom belongs to $\mathrm{CO}$ in a gas form. The prevalence of $\mathrm{CO}_{2}$ ice, which is mostly formed on grains, stresses the necessity of proper surface chemistry treatment.

In Figure 4, we present the distribution of important C-containing molecules over the disk for a model with typical parameters $\left(M_{\text {disk }}=0.01 M_{\odot}, M_{\star}=1.0 M_{\odot}, R_{\mathrm{c}}=100 \mathrm{au}\right.$, and $\gamma=0.8)$. Despite the CO snowline being expected to be located at $\sim 70 \mathrm{au}$, where the temperature reaches the critical value of $20 \mathrm{~K}$, we see a lack of gaseous $\mathrm{CO}$ in the disk midplane as close as $\sim 15$ au to the star. Even within the CO snowline in a region with temperature just above $20 \mathrm{~K}$, there is a constant exchange of $\mathrm{CO}$ molecules between gas and ice reservoirs. Here, some of the temporally frozen-out $\mathrm{CO}$ molecules are apparently converted into $\mathrm{CO}_{2}$ ice before they go back to the gas. The black and gray contours in Figure 4 indicate the area, limited by the criteria outlined by Williams \& Best (2014): CO is supposed to reside in the gas phase everywhere, except for the regions where it is frozen out $(T<20 \mathrm{~K})$ or photodissociated $\left(\Sigma_{\mathrm{H}_{2}}<1.3 \times 10^{21} \mathrm{~cm}^{-2}\right)$. But in our model, $\mathrm{CO}$ is not only highly depleted inside the restricted area due to chemical redistribution into other molecules, but it is also present outside of this area. Specifically, we see some CO in the outer disk region, which is shadowed from the star. In this cold region, $\mathrm{CO}$ is photodesorbed from dust grains, but not photodissociated by interstellar UV due to $\mathrm{H}_{2}$ shielding. This allows $\mathrm{CO}$ to be present in the gas phase despite temperatures below the freezing point.

In our set of models, the average total abundance ratio $\mathrm{iCO}_{2} / \mathrm{iH}_{2} \mathrm{O}$ is $1 / 3$. For the model shown in Figure 4 , the $\mathrm{iCO}_{2} / \mathrm{iH}_{2} \mathrm{O}$ abundance ratio varies with radius from $10 \%$ to $110 \%$ in the $6-120$ au range with a maximum at 20 au. CO ice dominates over $\mathrm{CO}_{2}$ ice beyond 200 au with a maximum abundance ratio relative to water of $60 \%$ at $300 \mathrm{au}$. These characteristic abundance ratios are consistent with what is observed in most comets (Mumma \& Charnley 2011). However, comparing our results to cometary abundances, one has to keep in mind that the abundances of molecules observed in comets depend also on nucleus structures and their thermal evolution (e.g., Hässig et al. 2015) and on the molecule processing in comae. The dynamical evolution of the comet ensemble is also of importance. In our model, this ratio is computed for a wide range of models and may differ from the corresponding ratios in specific models and/or spatial locations. 

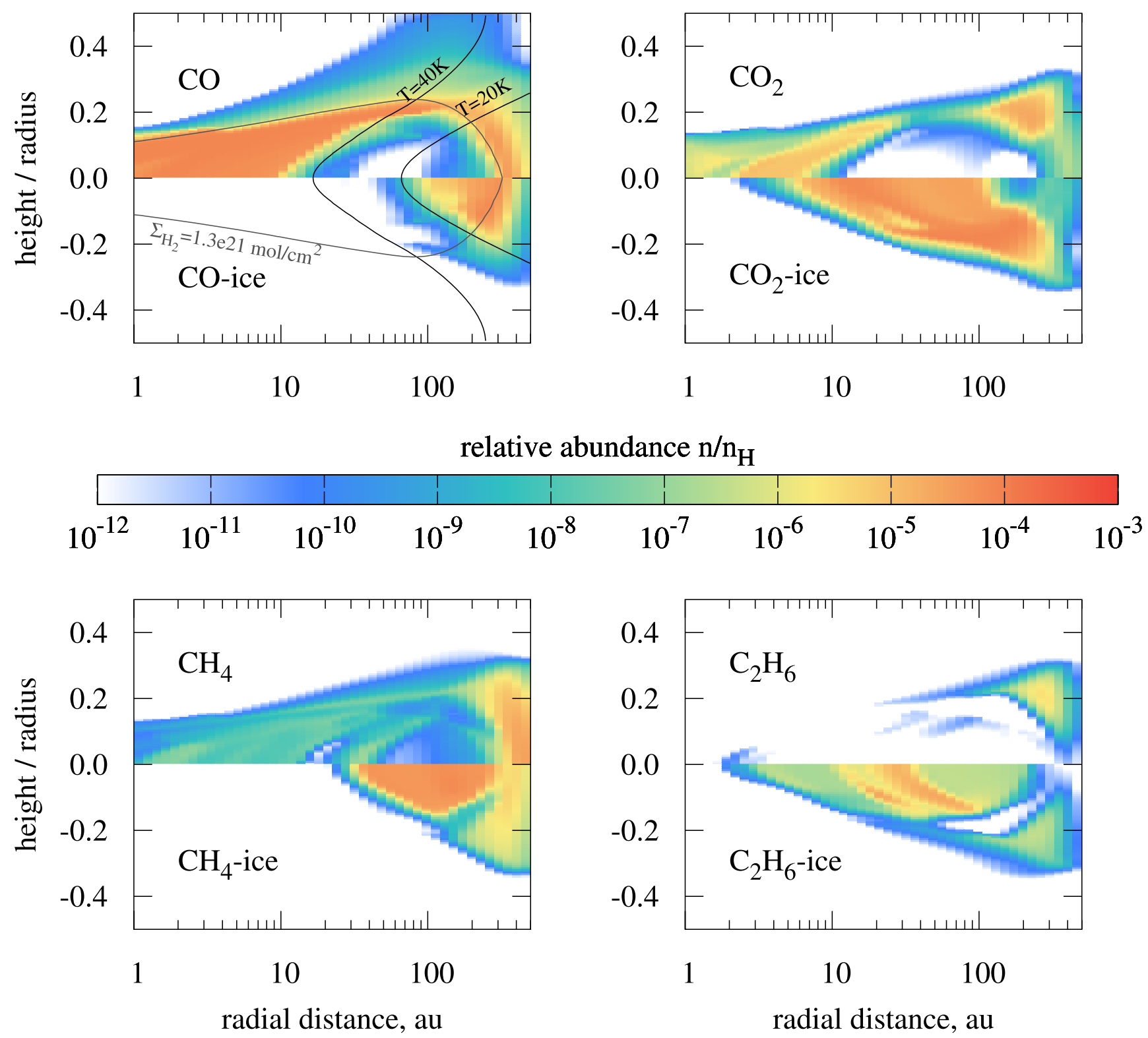

Figure 4. Abundances of major carbon-bearing species over the disk at the age of $3 \mathrm{Myr}$ for one particular model $\left(M_{\mathrm{disk}}=0.01 M_{\odot}, M_{\star}=1.0 M_{\odot}, R_{\mathrm{c}}=100\right.$ au, and $\gamma=0.8$ ). The upper half of the plots corresponds to gas abundances, while the bottom half shows the ice abundances.

The $\mathrm{CO}_{2}$ snowline is clearly seen at $\sim 6$ au, and a significant amount of $\mathrm{CO}_{2}$ ice resides in the dark disk regions up to distances of about 300 au from the star. Another abundant ice, methane, has a snowline at about 20-30 au from the star and is quite abundant in the midplane from 30 to 300 au. The region of abundant ethane ice extends down to a few astronomical units to the star, while in the gas phase this molecule is nearly absent. Overall, among all of the carbon-bearing species, $\mathrm{CO}$ is not the most abundant, but it is the only species that does not reside mostly in ices, but in the gas phase.

Lines defined by the above criteria delineate the part of the disk that contains $56 \%$ of its mass for the presented model. In other disks from our sample, this value changes from less than $5 \%$ in extremely low-mass disks transparent to UV photons and up to $94 \%$ in warm compact disks, with the mean value of about $50 \%$ over the whole ensemble. If the ISM-like CO abundance of $10^{-4}$ relative to $\mathrm{H}_{2}$ were present everywhere in the disk, and assuming that $\mathrm{CO}$ traces on average only $50 \%$ of the disk mass, we would get the $\mathrm{CO}$-average abundance in disk equal to $5 \times 10^{-5}$. On the other hand, our chemical modeling suggests $\overline{X_{\mathrm{CO}}}=8 \times 10^{-6}$ (see Table 3 ). Thus, we conclude that the $\mathrm{CO} / \mathrm{H}_{2}$ abundance ratio of $1.6 \times 10^{-5}$ should be used to determine the disk mass from $\mathrm{CO}$ observations, and that qualitative criteria of $\mathrm{CO}$ depletion overestimate its presence in the disk by a factor of 3 .

Another way to improve the Williams \& Best (2014) formalism would be to apply a different value of critical temperature, below which $\mathrm{CO}$ is absent in the gas phase. It can be seen from Figure 4 that the abundance of $\mathrm{CO}$ does reach $10^{-4}$ in the part of the disk where it is predominantly in the gas phase. The photodissociation limit is traced as well; it only misses gaseous $\mathrm{CO}$ in the region of low density in the disk atmosphere far from the star, which makes an order of a few percent contribution to the total CO mass. However, the line of 


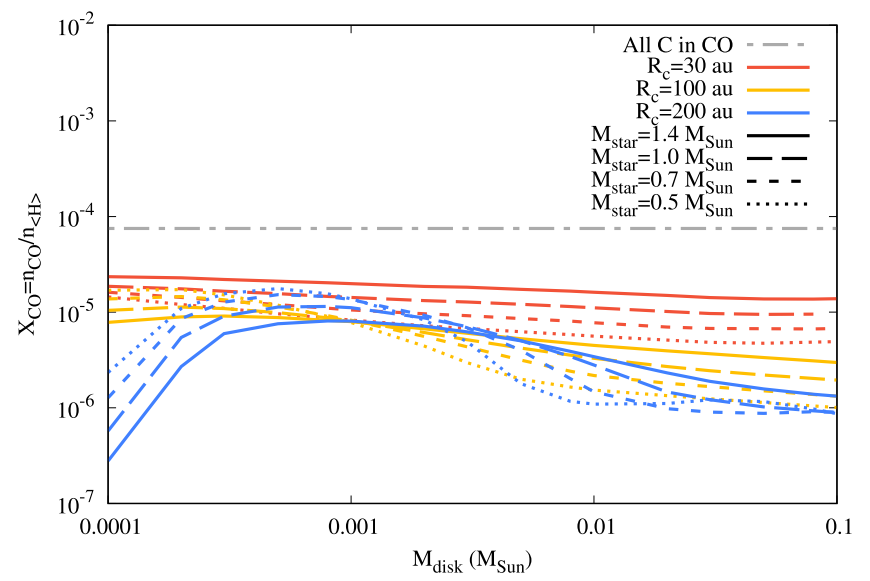

Figure 5. Total disk abundance of $\mathrm{CO}$ in disks of different characteristic radii $R_{\mathrm{c}}$ and around stars of different masses, $\gamma=0.5$, at the age of 3 Myr. Line color indicates $R_{\mathrm{c}}$, line styles (solid, dashed and dotted) indicate stellar mass. Maximum $X_{\mathrm{CO}}$ is shown with the gray line.

$\mathrm{CO}$ freeze-out defining the $\mathrm{CO}$ gas region should be replaced by the chemical depletion front, coincident with the $T \approx 40 \mathrm{~K}$ isoline (see Figure 4).

\subsection{Role of Individual Parameters}

In this section, we use the grid of models to investigate how the various parameters influence the $\mathrm{CO}$ abundance in the disk individually. For that purpose, we fix some parameters and check how the results change due to variations in other parameters.

Figure 5 illustrates the influence of the disk radius and the stellar mass on the scatter of $\mathrm{CO}$ total abundance at a fixed value $\gamma=0.5$. The star mass has only a small impact on the derived $\mathrm{CO}$ abundance in compact disks $\left(R_{\mathrm{c}}=30 \mathrm{au}\right)$. When we vary $M_{\star}$ between 0.5 and $1.4 M_{\odot}$, the CO total abundance in compact disks varies only by about a factor of 3 , and the higher the stellar mass, the larger the $\mathrm{CO}$ abundance. The influence of the stellar mass becomes more dramatic in disks of larger radii, at least, at the lower end of the considered disk mass range. Here, the same variation in $M_{\star}$ leads to an order of magnitude difference in $X_{\mathrm{CO}}$. The overall uncertainty of the $\mathrm{CO}$ content reaches two orders of magnitude in the least massive disks.

It is also interesting to note that in models with lower disk masses, a hotter star leads to a smaller $\mathrm{CO}$ abundance, while in models with higher disk masses, the opposite trend is observed. Obviously, the CO abundance is limited by photodissociation and photodesorption, and in various situations, the higher stellar temperature can lead to either a decrease or increase in the gas-phase $\mathrm{CO}$ abundance. Very low-mass disks, being more transparent to stellar UV radiation, suffer from CO photodestruction, which is stronger in disks around more massive stars. Thus, these disks contain less gas-phase CO. Low-mass disks with $R_{\mathrm{c}}=30$ au do not show this trend because they encompass the same mass in a more compact region, which results in higher densities and lower influence of photodissociation. In massive disks, photodissociation is not that crucial and $\mathrm{CO}$ depletion is mostly caused by chemical redistribution into ices, especially $\mathrm{CO}_{2}$ ice. A less massive star causes a $\mathrm{CO}_{2}$ snowline closer to the star, expanding the zone where $\mathrm{CO}$ is effectively removed by chemical processes.

Other considered values of $\gamma(0.8,1.0$ and 1.5) do not produce such a high variance in $\mathrm{CO}$ total abundance, with a larger $\gamma$ giving

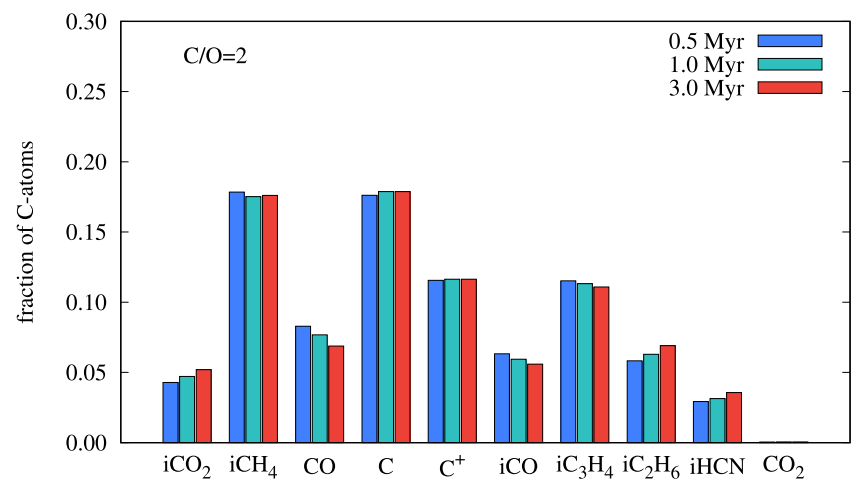

Figure 6. Same as Figure 3, but for $\mathrm{C} / \mathrm{O}=2$.

a more stable result. The differences described above are the highest in disks with $\gamma=0.5$, where the surface density falls off slowly, leaving more material in the outer regions of the disk, where $\mathrm{CO}$ depletion is stronger. The dependence on $R_{\mathrm{c}}$ is caused by a similar effect: disks of equal mass have more matter in the warm inner region if their radii are small, allowing more $\mathrm{CO}$ to reside in the gas phase. When $R_{\mathrm{c}}$ is large, more mass is in the cold outer area with high $\mathrm{CO}$ depletion.

\subsection{Initial $\mathrm{C} / \mathrm{O}$ Ratio}

The spatial distribution of carbon described in Section 3.2 was computed for a disk with low-metals initial abundance (Table 1), where there is more oxygen than carbon $(\mathrm{C} / \mathrm{O} \approx 0.4)$. To test the effect of other possible elemental compositions, we run 200 models, reducing the initial $\mathrm{O}$ abundance from $1.76 \times 10^{-4}$ to $3.65 \times 10^{-5}$ (leading to $\mathrm{C} / \mathrm{O}=2)$.

Figure 6 presents the fraction of carbon atoms locked up in the various species dominant in the disk at the previous value of $\mathrm{C} / \mathrm{O}$. Compared to Figure 3, the abundances of $\mathrm{CO}_{2}$ and $\mathrm{CO}$ both in the gas and ice dropped significantly because of the lack of oxygen. On average, only $\sim 7 \%$ of $\mathrm{C}$ is now in $\mathrm{CO}$ gas, while the amount of $\mathrm{CO}$ ice stays nearly the same.

Instead of $\mathrm{CO}_{2}$ ice, we have a richer carbon chemistry on dust surfaces. In addition to ices $\mathrm{iCH}_{4}, \mathrm{iC}_{2} \mathrm{H}_{6}$, and $\mathrm{iC}_{3} \mathrm{H}_{4}$, we end up with much higher abundances of molecules like $\mathrm{iC}_{5} \mathrm{H}_{2}$, $\mathrm{iCH}_{3} \mathrm{CN}, \mathrm{iC}_{9} \mathrm{H}_{2}, \mathrm{iCH}_{3} \mathrm{C}_{3} \mathrm{~N}$, and many other surface species with long $C$ chains (Herbst \& van Dishoeck 2009). Abundances of atomic and ionized carbon are also higher compared to the case of $\mathrm{C} / \mathrm{O}=0.4$.

Overall, the carbon distribution among species and the abundance of $\mathrm{CO}$ depend on the initial elemental composition as well.

For the present set of models, we calculated the scatter parameter $s$ as well. The $\mathrm{CO}$ scatter parameter is still close to the top with the value of 0.22 ; it follows $\mathrm{N}_{2}$ with $s=0.20$ and a few minor species having the same values of scatter but low mean abundances $\left(<10^{-9}\right)$. For this elemental composition, $\mathrm{CO}_{2}$, previously showing a low scatter, has $s=0.60$, which worsens its applicability as a mass tracer. Among the top species, only $\mathrm{CO}$ and $\mathrm{N}_{2}$ retain approximately the same values of scatter at $\mathrm{C} / \mathrm{O}=2$.

\section{Discussion}

Although observations of molecular lines remain the only tool to determine gas masses of protoplanetary disks, their interpretation is far from being easy. Even though the most common mass 
tracer in the interstellar and circumstellar medium, $\mathrm{CO}$, is believed to be controlled by a relatively simple set of processes, its straightforward application to gas mass determination in protoplanetary disks has produced results, which contradict both dust observations and HD observations (Bergin et al. 2013; Favre et al. 2013; McClure et al. 2016). The discrepancy between dustderived and CO-derived disk masses can in principle be explained by the dust evolution and gas dispersal, but it is harder to reconcile data on various molecular gas mass tracers, like $\mathrm{CO}$ and $\mathrm{HD}$, although this difference can be reduced by accounting for the $\mathrm{CO}$ isotope selection and carbon underabundance in disks, as Trapman et al. (2017) suggest.

The basic assumption behind the possibility of using $\mathrm{CO}$ as a mass tracer is that its abundance in protoplanetary disks is defined by the balance between photodissociation and freezeout. Williams \& Best (2014) suggested a grid of models based on the assumption that $\mathrm{CO}$ is frozen out everywhere, where the temperature is below $20 \mathrm{~K}$, and is photodissociated above the $\mathrm{H}_{2}$ column density of $1.3 \times 10^{21} \mathrm{~cm}^{-2}$. If none of these conditions is met, CO is assumed to have the "interstellar" abundance of $10^{-4}$. It becomes increasingly clear that simple prescriptions, like the one suggested by Williams \& Best (2014), can produce spurious results. First, as Williams \& Best (2014) already noted, more efficient depletion pathways are possible rather than simple $\mathrm{CO}$ ice formation. Also, the $\mathrm{CO}$ sublimation temperature depends on pressure, and under conditions of the disk midplane could be higher than $20 \mathrm{~K}$ (Harsono et al. 2015). ${ }^{6}$ Second, a typical "interstellar" CO abundance (inherited by the disks) can be lower than $10^{-4}$ (Burgh et al. 2007). One way to infer the $\mathrm{CO} / \mathrm{H}_{2}$ ratio in protoplanetary disks directly is to observe the absorption lines of both components in the UV band (France et al. 2014). This can be one of the prospects for future space UV missions like WSO-Spectrum UV (Boyarchuk et al. 2016) and LUVOIR. ${ }^{7}$

Obviously, more sophisticated methods should be used to infer the total gas mass from $\mathrm{CO}$ (or other molecule) observations. In a number of recent papers, the utility of $\mathrm{CO}$ as a mass tracer has been assessed by means of detailed models of the disk's physical and chemical structure. In a series of works by Miotello et al. (2014, 2016, 2017), a disk chemical model with isotope-selective processes was considered in order to find a way to determine the disk mass from $\mathrm{CO}$ isotopologue line observations. A large set of disk models was employed to study the evolution of ${ }^{13} \mathrm{CO}, \mathrm{C}^{17} \mathrm{O}$, and $\mathrm{C}^{18} \mathrm{O}$ in these disks. It was concluded that $\mathrm{CO}$ observations can lead to gas masses being underestimated in those disks where dust grains have grown to larger sizes. Application of the results to real observations produced very low gas masses in T Tauri disks, often lower than $1 M_{\mathrm{J}}$. It was noted that this can be an effect of sequestering carbon atoms in more complex molecules. This option has not been considered in the above study, as the chemical model used included only a limited set of surface processes (simple hydrogenation processes). The consequence can be seen in Figure 2 from Miotello et al. (2014), where the CO depletion zone is delineated by a $T=20 \mathrm{~K}$ line and the $\mathrm{CO}$ snowline is located at about $100 \mathrm{au}$.

A more detailed consideration of surface processes was performed by Reboussin et al. (2015). A single surface density

\footnotetext{
6 There is also evidence for the opposite. In some cases, $\mathrm{CO}$ in the midplane is detected at temperatures as low as $13 \mathrm{~K}$ (Dartois et al. 2003), which, probably, can be explained by turbulent mixing (Semenov et al. 2006).

https://asd.gsfc.nasa.gov/luvoir/
}

distribution was considered in this study, but with different vertical temperature profiles. It was shown that the surface conversion of $\mathrm{CO}$ into other ices, primarily $\mathrm{CO}_{2}$ ice, leads to gasphase $\mathrm{CO}$ abundances much lower than the canonical value of $10^{-4}$. Abundances that high are only reached in relatively warm disks with midplane temperatures above $30 \mathrm{~K}$ beyond $R=100$ au. In this work, results were presented only for the outer part of the disk $(R=100$ and $300 \mathrm{au})$, where theoretical CO abundance can be quite sensitive to details of RT (see our results above and also observations presented in Huang et al. 2016).

A chemical evolution with a detailed account of surface reactions and carbon isotope-selective processes was considered by $\mathrm{Yu}$ et al. (2016, 2017). They studied the chemical evolution of a typical disk within the inner 70 au and found that due to chemical depletion, i.e., the conversion of $\mathrm{CO}$ into less volatile molecules, the effective $\mathrm{CO}$ snowline is located at about $20 \mathrm{au}$ and moves toward the star as the disk evolves, even though the temperature in their model is above the $\mathrm{CO}$ sublimation point everywhere in the considered region. According to their results, after $3 \mathrm{Myr}$ of evolution, $13.6 \%$ of all available carbon is locked in gas-phase $\mathrm{CO}$, while $\mathrm{CO}_{2}$ ice contains $36.2 \%$ of carbon atoms, which compares favorably with our results for a similar disk model (we must note that this agreement is reached despite quite different assumptions on dust properties). Although the average value of carbon partition matches well, its dispersion is significant in our modeling (from a few percent up to $50 \%$ ). They also found that the $\mathrm{CO}$ abundance varies with time significantly, which is something that we do not see in our model. However, in their model, they vary stellar properties with time, while we keep them constant. The overall conclusion of $\mathrm{Yu}$ et al. (2017) is that a straightforward interpretation of $\mathrm{CO}$ observations leads to the underestimation of disk masses. Thus, all of the conclusions on the gas deficit in protoplanetary disks based on $\mathrm{CO}$ observations should be considered with caution.

The focus of the above studies was on isotope-selective processes and on the use of $\mathrm{CO}$ isotopologue line ratios as mass indicators. In our study, we consider $\mathrm{CO}$ as a whole, without distinguishing among its isotopologues, concentrating rather on the global chemical aspects of $\mathrm{CO}$ distributions in disks having different structural parameters.

In our study, we consider a wide range of disk sizes, masses, and surface density profiles, assuming that there is no dust evolution, so that the dust still has its interstellar parameters. Our results indicate that $\mathrm{CO}$ is not an ideal mass tracer, but all other molecules show greater uncertainties in the relative abundance. Still, some of them, e.g., $\mathrm{H}_{2} \mathrm{O}, \mathrm{H}_{2} \mathrm{CO}$, and especially $\mathrm{CO}_{2}$, can be used as supplementary tracers, particularly if some information on the disk structure are available.

A better calibration of $\mathrm{CO}$ observations can be obtained using the Williams \& Best (2014) method, assuming a factor of a few lower typical abundance and taking into account the chemical depletion (e.g., taking $40 \mathrm{~K}$ as the $\mathrm{CO}$ depletion temperature).

An obvious extension of the study is to consider the dependence of our results on the assumed grain size as dust is expected to grow in protoplanetary disks. Akimkin et al. (2013) have shown that the effect of grain growth at its initial stage is to shift the molecular layer closer to the dense midplane. We may provisionally expect that this is going to make molecules better gas mass tracers, but this is a subject for future study. 
Table 4

Average Total Abundance $\overline{X_{i}}=10^{\overline{x_{i}}}$ and Scatter $s_{i}$ for Ice Species

\begin{tabular}{|c|c|c|c|c|c|c|c|c|}
\hline Species & $\overline{X_{i}}$ & $s_{i}(\operatorname{dex})$ & Species & $\overline{\overline{X_{i}}}$ & $s_{i}(\operatorname{dex})$ & Species & $\overline{X_{i}}$ & $s_{i}(\operatorname{dex})$ \\
\hline iSiO & $8.1 \times 10^{-10}$ & 0.20 & $\mathrm{iCH}_{3} \mathrm{CN}$ & $1.1 \times 10^{-9}$ & 0.62 & $\mathrm{iC}_{6} \mathrm{H}_{6}$ & $2.1 \times 10^{-11}$ & 0.94 \\
\hline $\mathrm{iCO}_{2}$ & $1.7 \times 10^{-5}$ & 0.26 & $\mathrm{iC}_{3} \mathrm{H}_{4}$ & $6.7 \times 10^{-7}$ & 0.65 & $\mathrm{iH}_{2} \mathrm{~S}_{2}$ & $5.1 \times 10^{-10}$ & 0.96 \\
\hline $\mathrm{iFeH}$ & $1.9 \times 10^{-9}$ & 0.26 & iPO & $1.2 \times 10^{-11}$ & 0.65 & $\mathrm{iH}_{2} \mathrm{CO}$ & $6.6 \times 10^{-8}$ & 0.96 \\
\hline $\mathrm{iMgH}_{2}$ & $4.4 \times 10^{-9}$ & 0.28 & $\mathrm{iHC}_{3} \mathrm{~N}$ & $2.1 \times 10^{-11}$ & 0.65 & $\mathrm{iH}_{2}$ & $1.1 \times 10^{-7}$ & 0.96 \\
\hline $\mathrm{iC}_{2} \mathrm{H}_{4}$ & $3.0 \times 10^{-10}$ & 0.28 & $\mathrm{iC}_{7} \mathrm{H}_{2}$ & $1.9 \times 10^{-11}$ & 0.66 & $\mathrm{iH}_{2} \mathrm{C}_{3} \mathrm{O}$ & $4.6 \times 10^{-10}$ & 0.99 \\
\hline $\mathrm{iHNC}$ & $1.1 \times 10^{-8}$ & 0.29 & $\mathrm{iC}_{8} \mathrm{H}_{4}$ & $1.0 \times 10^{-9}$ & 0.66 & $\mathrm{iCH}_{3} \mathrm{C}_{4} \mathrm{H}$ & $8.0 \times 10^{-11}$ & 1.05 \\
\hline iHNCO & $3.5 \times 10^{-9}$ & 0.34 & $\mathrm{iC}_{4} \mathrm{~N}$ & $1.5 \times 10^{-11}$ & 0.67 & $\mathrm{iNH}_{2} \mathrm{CHO}$ & $2.3 \times 10^{-9}$ & 1.10 \\
\hline $\mathrm{iSiH}_{4}$ & $3.8 \times 10^{-9}$ & 0.35 & $\mathrm{iH}_{3} \mathrm{C}_{5} \mathrm{~N}$ & $3.2 \times 10^{-11}$ & 0.69 & $\mathrm{iCH}_{2} \mathrm{CO}$ & $1.7 \times 10^{-9}$ & 1.17 \\
\hline iP & $7.6 \times 10^{-11}$ & 0.36 & $\mathrm{iCH}_{3} \mathrm{OH}$ & $5.2 \times 10^{-8}$ & 0.69 & iHNO & $3.3 \times 10^{-9}$ & 1.29 \\
\hline $\mathrm{iHCN}$ & $9.8 \times 10^{-7}$ & 0.37 & $\mathrm{iC}_{8} \mathrm{H}_{2}$ & $1.6 \times 10^{-11}$ & 0.70 & $\mathrm{iO}_{2}$ & $7.6 \times 10^{-10}$ & 1.32 \\
\hline $\mathrm{iHCl}$ & $2.4 \times 10^{-10}$ & 0.38 & $\mathrm{iH}_{5} \mathrm{C}_{3} \mathrm{~N}$ & $2.2 \times 10^{-10}$ & 0.71 & $\mathrm{iS}_{2}$ & $4.0 \times 10^{-10}$ & 1.38 \\
\hline $\mathrm{iC}_{5} \mathrm{H}_{2}$ & $3.3 \times 10^{-10}$ & 0.39 & $\mathrm{iC}_{7} \mathrm{H}_{4}$ & $1.9 \times 10^{-9}$ & 0.72 & $\mathrm{iNH}_{2}$ & $2.3 \times 10^{-11}$ & 1.39 \\
\hline $\mathrm{iCl}$ & $2.9 \times 10^{-10}$ & 0.40 & $\mathrm{iCH}_{4}$ & $5.3 \times 10^{-6}$ & 0.73 & iNO & $1.1 \times 10^{-11}$ & 1.49 \\
\hline $\mathrm{iH}_{2} \mathrm{O}$ & $5.1 \times 10^{-5}$ & 0.40 & $\mathrm{iC}_{5} \mathrm{H}_{4}$ & $7.6 \times 10^{-8}$ & 0.73 & $\mathrm{iC}_{4} \mathrm{H}_{4}$ & $4.3 \times 10^{-10}$ & 1.50 \\
\hline $\mathrm{iNaH}$ & $9.5 \times 10^{-10}$ & 0.43 & $\mathrm{iC}_{2} \mathrm{H}_{6}$ & $7.3 \times 10^{-7}$ & 0.74 & $\mathrm{iCH}_{3} \mathrm{OCH}_{3}$ & $1.9 \times 10^{-11}$ & 1.52 \\
\hline $\mathrm{iNH}_{3}$ & $6.6 \times 10^{-6}$ & 0.46 & $\mathrm{iN}_{2} \mathrm{H}_{2}$ & $2.3 \times 10^{-11}$ & 0.76 & $\mathrm{iC}_{2} \mathrm{H}_{5} \mathrm{OH}$ & $2.0 \times 10^{-11}$ & 1.53 \\
\hline $\mathrm{iH}_{2} \mathrm{O}_{2}$ & $5.2 \times 10^{-8}$ & 0.49 & $\mathrm{iH}_{2} \mathrm{CS}$ & $4.5 \times 10^{-9}$ & 0.81 & $\mathrm{iOH}$ & $5.0 \times 10^{-10}$ & 1.56 \\
\hline $\mathrm{iC}_{3} \mathrm{~S}$ & $7.3 \times 10^{-11}$ & 0.49 & $\mathrm{iHCOOH}$ & $1.6 \times 10^{-8}$ & 0.82 & $\mathrm{iCH}_{3} \mathrm{CHO}$ & $2.3 \times 10^{-9}$ & 1.57 \\
\hline $\mathrm{iC}_{6} \mathrm{H}_{2}$ & $9.4 \times 10^{-11}$ & 0.49 & $\mathrm{iC}_{9} \mathrm{H}_{4}$ & $2.2 \times 10^{-9}$ & 0.83 & $\mathrm{iCH}_{3}$ & $3.1 \times 10^{-11}$ & 1.60 \\
\hline $\mathrm{iNH}_{2} \mathrm{OH}$ & $7.8 \times 10^{-10}$ & 0.53 & $\mathrm{iCH}_{5} \mathrm{~N}$ & $9.6 \times 10^{-9}$ & 0.83 & $\mathrm{iCH}_{2}$ & $3.0 \times 10^{-11}$ & 1.64 \\
\hline $\mathrm{iN}_{2}$ & $6.8 \times 10^{-7}$ & 0.55 & $\mathrm{iC}_{9} \mathrm{H}_{2}$ & $6.3 \times 10^{-11}$ & 0.84 & $\mathrm{iCH}$ & $2.2 \times 10^{-11}$ & 1.75 \\
\hline $\mathrm{iCO}$ & $3.2 \times 10^{-6}$ & 0.58 & $\mathrm{iO}_{3}$ & $2.7 \times 10^{-9}$ & 0.85 & $\mathrm{iO}$ & $1.3 \times 10^{-10}$ & 1.75 \\
\hline $\mathrm{iH}_{2} \mathrm{~S}$ & $2.3 \times 10^{-8}$ & 0.59 & $\mathrm{iHS}_{2}$ & $2.3 \times 10^{-10}$ & 0.86 & $\mathrm{iC}$ & $2.2 \times 10^{-11}$ & 1.86 \\
\hline $\mathrm{iC}_{3} \mathrm{H}_{2}$ & $1.2 \times 10^{-9}$ & 0.60 & $\mathrm{iC}_{6} \mathrm{H}_{4}$ & $1.8 \times 10^{-8}$ & 0.88 & $\mathrm{iH}$ & $2.4 \times 10^{-9}$ & 1.98 \\
\hline $\mathrm{iC}_{2} \mathrm{H}_{2}$ & $9.5 \times 10^{-11}$ & 0.61 & $\mathrm{iN}_{2} \mathrm{O}$ & $1.1 \times 10^{-11}$ & 0.92 & & & \\
\hline
\end{tabular}

\section{Conclusions}

We conducted a chemical modeling of $\sim 1000$ protoplanetary disk structures around low-mass stars with different parameters to find out species that have the best correlation with the total disk mass. We varied the central star mass, the disk characteristic radius, the disk mass, and the $\gamma$ index of the surface density distribution, assuming MRN grain size distribution for the UV RT in the disk atmosphere and $0.1 \mu \mathrm{m}$ size grains for the surface chemistry. The chemical modeling is based on the updated non-equilibrium chemical network ALCHEMIC (Semenov \& Wiebe 2011) incorporated into the ANDES code (Akimkin et al. 2013). We focused on an intrinsic dispersion of species abundance due to unknown structural and thermal parameters. The main conclusions are:

1. Among all 650 considered species, the relative abundance of the $\mathrm{CO}$ molecule has one of the smallest scatters in the overall disk ensemble (with the obvious exceptions of hydrogen and helium). The characteristic reference " $1 \sigma$ " values for the logarithm of the species total disk abundance at an age of $3 \mathrm{Myr}$ are $0.28 \mathrm{dex}$ for $\mathrm{CO}$ and $\mathrm{CO}_{2}, 0.29$ dex for $\mathrm{H}_{2} \mathrm{CO}$, and 0.51 dex for $\mathrm{H}_{2} \mathrm{O}$ (see Table 3 for other species). So, even in the case of $\mathrm{CO}$, there is a maximum uncertainty of one order of magnitude in $\mathrm{CO}$ abundance if the disk physical structure is unknown. More reliable CO disk masses can be obtained if the disk characteristic radius $R_{\mathrm{c}}$ is determined.

2 . On average, in the whole disk ensemble, $\approx 13 \%$ of carbon atoms end up in the gas-phase $\mathrm{CO}$ molecule (for the initial "low-metals" abundance from Lee et al. 1998 where $\mathrm{C} / \mathrm{O}=0.4$ ). The average value of carbon partitioning is in good agreement with the conclusions by $\mathrm{Yu}$ et al. (2016). However, the extreme values vary from $\approx 2 \%$ for large and massive disks to $\approx 30 \%$ for compact disks with small and moderate masses. Statistically, the most abundant carbonbearing species is $\mathrm{CO}_{2}$ ice, with an average $\mathrm{C}$ atom fraction of $\approx 25 \%$. The majority of the remaining carbon atoms are in $\mathrm{CH}_{4}$ and $\mathrm{CO}$ ices (17\% and $7 \%$ of $\mathrm{C}$ atoms, respectively), $\mathrm{C}$ and $\mathrm{C}^{+}$ (14\% and $8 \%$ ), and ices of complex organic species. The degree of carbon atom redistribution into non- $\mathrm{CO}$ molecules is naturally more effective if $\mathrm{C} / \mathrm{O}>1$. Quantitatively, the average carbon partition into $\mathrm{CO}$ is $7 \%$ for $\mathrm{C} / \mathrm{O}=2$.

3. Despite $\mathrm{CO}$ having a noticeable variance in the relative abundance, it is still the best molecular tracer of disk gas mass. The typical value of the total $\mathrm{CO} / \mathrm{H}_{2}$ abundance ratio is $1.6 \times 10^{-5}$ with " $1 \sigma$ " limits from $0.8 \times 10^{-5}$ to $3.0 \times 10^{-5}$. $\mathrm{CO}_{2}$ also has a low abundance variance, although this variance depends strongly on the $\mathrm{C} / \mathrm{O}$ ratio. Other species that have relatively good correlation with disk mass are $\mathrm{H}_{2} \mathrm{O}$ and $\mathrm{H}_{2} \mathrm{CO}$.

We find that on average the total abundance of gaseous $\mathrm{CO}$ in protoplanetary disks is $\approx 6$ times lower compared to the interstellar value of $10^{-4}$. Chemical depletion lowers the abundance of $\mathrm{CO}$ by a factor of 3, compared to the case of photodissociation and freeze-out as the only ways of $\mathrm{CO}$ destruction.

We thank the referee for fruitful comments that helped us improve our model and formulate better conclusions. T.M., V.A., and D.W. acknowledge financial support from the Russian Science Foundation (17-12-01441; Sections 2, 3, 5). D.S. acknowledges support from the Heidelberg Institute of Theoretical Studies for the project "Chemical kinetics models and visualization tools: Bridging biology and astronomy." A.V. acknowledges support from the European Research Council (ERC; project PALs 320620).

\section{Appendix Ice Species}

Here we present a version of Table 3 for ice species. Table 4 lists ice species with average total abundance in the disk above $10^{-11}$ sorted by scatter. 


\section{ORCID iDs}

Tamara Molyarova (1) https://orcid.org/0000-0003-0448-6354 Vitaly Akimkin (1) https://orcid.org/0000-0002-4324-3809

Anton Vasyunin (16) https://orcid.org/0000-0003-1684-3355

Dmitri Wiebe (1) https://orcid.org/0000-0002-5111-0395

\section{References}

Akimkin, V., Zhukovska, S., Wiebe, D., et al. 2013, ApJ, 766, 8 Akimkin, V. V. 2015, ARep, 59, 747

Andrews, S. M., \& Williams, J. P. 2005, ApJ, 631, 1134

Ansdell, M., Williams, J. P., Manara, C. F., et al. 2017, AJ, 153, 240

Ansdell, M., Williams, J. P., van der Marel, N., et al. 2016, ApJ, 828, 46

Armitage, P. J. 2015, arXiv:1509.06382

Bai, X.-N., \& Goodman, J. 2009, ApJ, 701, 737

Baraffe, I., Homeier, D., Allard, F., \& Chabrier, G. 2015, A\&A, 577, A42

Bergin, E. A., Cleeves, L. I., Gorti, U., et al. 2013, Natur, 493, 644

Bergin, E. A., Hogerheijde, M. R., Brinch, C., et al. 2010, A\&A, 521, L33

Bitsch, B., Lambrechts, M., \& Johansen, A. 2015, A\&A, 582, A112

Bohlin, R. C., Savage, B. D., \& Drake, J. F. 1978, ApJ, 224, 132

Bolatto, A. D., Wolfire, M., \& Leroy, A. K. 2013, ARA\&A, 51, 207

Boyarchuk, A. A., Shustov, B. M., Savanov, I. S., et al. 2016, ARep, 60, 1

Burgh, E. B., France, K., \& McCandliss, S. R. 2007, ApJ, 658, 446

Carmona, A., van der Plas, G., van den Ancker, M. E., et al. 2011, A\&A, 533, A39

Chapillon, E., Guilloteau, S., Dutrey, A., Piétu, V., \& Guélin, M. 2012, A\&A, 537, A60

Chapillon, E., Parise, B., Guilloteau, S., \& Du, F. 2011, A\&A, 533, A143

Chiang, E. I., \& Goldreich, P. 1997, ApJ, 490, 368

Dartois, E., Dutrey, A., \& Guilloteau, S. 2003, A\&A, 399, 773

Draine, B. T. 2006, ApJ, 636, 1114

Dullemond, C. P., \& Dominik, C. 2004, A\&A, 421, 1075

Dullemond, C. P., Dominik, C., \& Natta, A. 2001, ApJ, 560, 957

Dunham, M. M., Vorobyov, E. I., \& Arce, H. G. 2014, MNRAS, 444, 887

Dutrey, A., Guilloteau, S., \& Guelin, M. 1997, A\&A, 317, L55

Dutrey, A., Wakelam, V., Boehler, Y., et al. 2011, A\&A, 535, A104

Favre, C., Cleeves, L. I., Bergin, E. A., Qi, C., \& Blake, G. A. 2013, ApJL, 776, L38

France, K., Herczeg, G. J., McJunkin, M., \& Penton, S. V. 2014, ApJ, 794, 160

Goldsmith, P. F., Bergin, E. A., \& Lis, D. C. 1997, ApJ, 491, 615

Guilloteau, S., Reboussin, L., Dutrey, A., et al. 2016, A\&A, 592, A124

Harsono, D., Bruderer, S., \& van Dishoeck, E. F. 2015, A\&A, 582, A41

Hartmann, L., Calvet, N., Gullbring, E., \& D'Alessio, P. 1998, ApJ, 495, 385

Hässig, M., Altwegg, K., Balsiger, H., et al. 2015, Sci, 347, aaa0276

Henning, T., \& Meeus, G. 2011, in Dust Processing and Mineralogy in Protoplanetary Accretion Disks, ed. P. J. V. Garcia (Chicago, IL: Univ. Chicago Press), 114

Henning, T., \& Semenov, D. 2008, in IAU Symp. 251, Organic Matter in Space, ed. S. Kwok \& S. Sanford (Cambridge: Cambridge Univ. Press), 89
Henning, T., \& Semenov, D. 2013, ChRv, 113, 9016

Herbst, E., \& van Dishoeck, E. F. 2009, ARA\&A, 47, 427

Hogerheijde, M. R., Bergin, E. A., Brinch, C., et al. 2011, Sci, 334, 338

Huang, J., Öberg, K. I., \& Andrews, S. M. 2016, ApJL, 823, L18

Isella, A., Guidi, G., Testi, L., et al. 2016, PhRvL, 117, 251101

Ivlev, A. V., Akimkin, V. V., \& Caselli, P. 2016, ApJ, 833, 92

Laor, A., \& Draine, B. T. 1993, ApJ, 402, 441

Lee, H.-H., Roueff, E., Pineau des Forets, G., et al. 1998, A\&A, 334, 1047

Loomis, R. A., Cleeves, L. I., Öberg, K. I., Guzman, V. V., \& Andrews, S. M. 2015, ApJL, 809, L25

Mathis, J. S., Mezger, P. G., \& Panagia, N. 1983, A\&A, 128, 212

Mathis, J. S., Rumpl, W., \& Nordsieck, K. H. 1977, ApJ, 217, 425

McClure, M. K., Bergin, E. A., Cleeves, L. I., et al. 2016, ApJ, 831, 167

Miotello, A., Bruderer, S., \& van Dishoeck, E. F. 2014, A\&A, 572, A96

Miotello, A., van Dishoeck, E. F., Kama, M., \& Bruderer, S. 2016, A\&A, 594, A85

Miotello, A., van Dishoeck, E. F., Williams, J. P., et al. 2017, A\&A, 599, A113

Mordasini, C., Alibert, Y., Benz, W., Klahr, H., \& Henning, T. 2012, A\&A, 541, A97

Mumma, M. J., \& Charnley, S. B. 2011, ARA\&A, 49, 471

Öberg, K. I., Guzmán, V. V., Furuya, K., et al. 2015, Natur, 520, 198

Öberg, K. I., Guzmán, V. V., Merchantz, C. J., et al. 2017, ApJ, 839, 43

Okuzumi, S. 2009, ApJ, 698, 1122

Qi, C., D'Alessio, P., Öberg, K. I., et al. 2011, ApJ, 740, 84

Qi, C., Öberg, K. I., \& Wilner, D. J. 2013, ApJ, 765, 34

Rab, C., Elbakyan, V., Vorobyov, E., et al. 2017, A\&A, 604, A15

Reboussin, L., Wakelam, V., Guilloteau, S., Hersant, F., \& Dutrey, A. 2015, A\&A, 579, A82

Salinas, V. N., Hogerheijde, M. R., Bergin, E. A., et al. 2016, A\&A, 591, A122

Sano, T., Miyama, S. M., Umebayashi, T., \& Nakano, T. 2000, ApJ, 543, 486

Semenov, D., \& Wiebe, D. 2011, ApJS, 196, 25

Semenov, D., Wiebe, D., \& Henning, T. 2006, ApJL, 647, L57

Thi, W.-F., Ménard, F., Meeus, G., et al. 2011, A\&A, 530, L2

Trapman, L., Miotello, A., Kama, M., van Dishoeck, E. F., \& Bruderer, S. 2017, A\&A, 605, A69

Tsukamoto, Y., Okuzumi, S., \& Kataoka, A. 2017, ApJ, 838, 151

Vasyunin, A. I., Caselli, P., Dulieu, F., \& Jiménez-Serra, I. 2017, ApJ, 842, 33

Vasyunina, T., Vasyunin, A. I., Herbst, E., \& Linz, H. 2012, ApJ, 751, 105

Wakelam, V., Loison, J.-C., Herbst, E., et al. 2015, ApJS, 217, 20

Walsh, C., Loomis, R. A., Öberg, K. I., et al. 2016, ApJL, 823, L10

Williams, J. P., \& Best, W. M. J. 2014, ApJ, 788, 59

Williams, J. P., \& Cieza, L. A. 2011, ARA\&A, 49, 67

Williams, J. P., \& McPartland, C. 2016, ApJ, 830, 32

Yu, M., Evans, N. J., II, Dodson-Robinson, S. E., Willacy, K., \& Turner, N. J. 2017, ApJ, 841, 39

Yu, M., Willacy, K., Dodson-Robinson, S. E., Turner, N. J., \& Evans, N. J., II 2016, ApJ, 822, 53

Zhang, K., Bergin, E. A., Blake, G. A., Cleeves, L. I., \& Schwarz, K. R. 2017, NatAs, 1, 0130 